\title{
Unanimous and strategy-proof probabilistic rules for single-peaked preference profiles on graphs
}

Citation for published version (APA):

Peters, H., Roy, S., \& Sadhukhan, S. (2021). Unanimous and strategy-proof probabilistic rules for singlepeaked preference profiles on graphs. Mathematics of Operations Research, 46(2), 811-833. https://doi.org/10.1287/moor.2020.1089

Document status and date:

Published: 01/05/2021

DOI:

10.1287/moor.2020.1089

Document Version:

Early version submitted to journal

\section{Please check the document version of this publication:}

- A submitted manuscript is the version of the article upon submission and before peer-review. There can be important differences between the submitted version and the official published version of record.

People interested in the research are advised to contact the author for the final version of the publication, or visit the DOI to the publisher's website.

- The final author version and the galley proof are versions of the publication after peer review.

- The final published version features the final layout of the paper including the volume, issue and page numbers.

Link to publication

\footnotetext{
General rights rights.

- You may freely distribute the URL identifying the publication in the public portal. please follow below link for the End User Agreement:

www.umlib.nl/taverne-license

Take down policy

If you believe that this document breaches copyright please contact us at:

repository@maastrichtuniversity.nl

providing details and we will investigate your claim.
}

Copyright and moral rights for the publications made accessible in the public portal are retained by the authors and/or other copyright owners and it is a condition of accessing publications that users recognise and abide by the legal requirements associated with these

- Users may download and print one copy of any publication from the public portal for the purpose of private study or research.

- You may not further distribute the material or use it for any profit-making activity or commercial gain

If the publication is distributed under the terms of Article $25 \mathrm{fa}$ of the Dutch Copyright Act, indicated by the "Taverne" license above, 


\title{
UNANIMOUS AND STRATEGY-PROOF PROBABILISTIC RULES FOR SINGLE-PEAKED PREFERENCE PROFILES ON GRAPHS*
}

\author{
Hans Peters ${ }^{\dagger} \quad$ Souvik Roy ${ }^{\ddagger} \quad$ Soumyarup Sadhukhan ${ }^{\S}$ \\ March 2019
}

\begin{abstract}
Finitely many agents have single-peaked preferences on a finite set of alternatives structured by a connected graph. First, all unanimous and strategy-proof probabilistic rules are characterized when the graph is a tree. These rules are uniquely determined by their outcomes at those preference profiles where all peaks are on leafs of the tree, and thus extend the known case of a line graph. Second, it is shown that every unanimous and strategy-proof probabilistic rule is random dictatorial if and only if the graph has no leafs. Finally, the two results are combined to obtain a general characterization for every connected graph.
\end{abstract}

JEL Classification: D71

Keywords: probabilistic rules, unanimity, single-peaked preferences, strategy-proofness, graphs

\section{Introduction}

Finitely many agents have preferences over a finite set of alternatives. The alternatives are the vertices in a connected graph, and the preferences of an agent are linear orderings which are single-peaked with respect to some spanning tree of the graph: there is a single top alternative, the peak, and preference decreases along the paths in this tree away from the peak. The objective is to choose an alternative based on these preferences, or rather - in this paper - a probability distribution over the alternatives.

An example of such a situation is a road or railroad network, where the vertices (junctions) are also the locations of villages or cities. The objective is to locate a public good (shopping mall, museum, hospital, school, etc.) based on the preferences of the agents over

*The authors would like to thank Alexander Grigoriev, Arunava Sen and Huaxia Zeng for helpful suggestions. This project has received funding from the European Union's Horizon 2020 research and innovation program under the Marie-Sklodowska-Curie grant agreement N0 660341.

${ }^{\dagger}$ Department of Quantitative Economics, School of Business and Economics, Maastricht University. Email: h.peters@maastrichtuniversity.nl

${ }^{\ddagger}$ Economic Research Unit, Indian Statistical Institute, Kolkata. Email: souvik. 2004@gmail.com

$\S$ Economic Research Unit, Indian Statistical Institute, Kolkata.

Email: soumyarup.sadhukhan@gmail.com 
these junctions. Distance from one's home or from a nearby bus stop may determine preference, but also the path one has to take. Single-peakedness is then a plausible assumption. Alternatively, the graph may represent a network of personal relations between the agents, and the objective is to distribute a public good - e.g., disperse information - over the vertices in this network. Also here, both the length of a path and the nodes (e.g., friends) to be visited may be important determinants for preference, and single-peakedness along a specific spanning tree captures this.

We consider probabilistic rules: these assign a probability distribution over the alternatives to every profile of single-peaked preferences. The conditions we impose are unanimity and strategy-proofness. Unanimity means that if all agents have the same peak then probability one is assigned to that alternative. Strategy-proofness means that no agent, by misrepresenting its true preference, can increase the probability on any upper contour set, i.e., any set of alternatives (weakly) preferred to some given alternative. Put differently, the probability distribution attained by reporting truthfully stochastically dominates any probability distribution achievable by misreporting.

We first consider the case where the graph has no cycles, i.e., is a tree (and thus its unique spanning tree). For this case, our main result (Theorem 3.13) is that a probabilistic rule is unanimous and strategy-proof if and only if it is 'monotonic'. In a nutshell, this means that such a rule is uniquely determined by the probability distributions it assigns to the preference profiles with all peaks at the leafs of the tree (i.e., the alternatives with degree one). We show that such a collection of probability distributions has the following properties: (i) a leaf is assigned probability one if all peaks are at this leaf; (ii) if an agent changes its peak from one leaf to another, then (a) probability does not decrease along the path from the former to the latter and (b) probability does not change off this path. These collections of probability distributions are also called 'monotonic'. They play a role similar to the collections of 'fixed probabilistic ballots' in Ehlers et al (2002) - see also below.

Second, for the case where the graph is arbitrary (but connected), we show that every unanimous and strategy-proof probabilistic rule is random dictatorial if and only if the graph has no leafs. In fact, we show this for the case of two agents and then extend the result to more than two agents by using a result of Chatterji et al (2014) - this is Theorem 4.7. Random dictatorship means that each agent is assigned a fixed probability (weight) and every alternative is chosen with probability equal to the sum of the probabilities of the agents having this alternative as their peak. If the graph is not a tree but has a leaf, then indeed unanimous and strategy-proof random social choice functions exist which are not random dictatorial, as we show by an example, and as also follows from the main result of the paper later on (Theorem 5.5). In order to prove Theorem 4.7 we first consider 2-connected graphs, i.e., graphs in which for every pair of distinct alternatives there is a cycle containing them, and next extend to arbitrary leafless graphs by decomposing the graph in a way analogous to the concept of a 'block tree' (Menger, 1927; Whitney, 1931; or, e.g., Bondy and Murty, 2008).

Third, for the general case, where the graph is not necessarily a tree, can have leafs, but is still connected, we show that every unanimous and strategy-proof probabilistic rule behaves like a monotonic rule on the branches of the graph and as a random dictatorial rule on the maximal leafless subgraph of the graph, such that the total probability on each branch is equal to the total weight of the agents who have their peaks on this branch. This is Theorem 5.5, which generalizes both Theorems 3.13 and 4.7.

Our first main result, Theorem 3.13 on trees, generalizes the case where the alternatives 
are ordered on a straight line and agents have single-peaked preferences. The latter case has been dealt with in Ehlers et al (2002): they consider the whole real line, but their characterization remains valid on a finite or discrete set of alternatives. In Peters et al (2014) it is shown that, for the version with finitely many alternatives, all probabilistic rules are convex combinations of deterministic rules. In the tree case it turns out that this no longer holds - see Section 6 for an example of a unanimous and strategy-proof probabilistic rule which is not a convex combination of deterministic rules with these properties. This supports the fact that the general tree case is not a straightforward generalization of the straight line case.

A consequence of Theorem 3.13 is a characterization of all unanimous and strategy-proof deterministic rules if agents have single-peaked preferences on a tree, which to the best of our knowledge is new as well (see Section 6). Schummer and Vohra (2002) also consider this issue but their setting is different: a graph is a subset of some Euclidean space (so there are infinitely many alternatives), and preferences are uniquely determined by their peaks by considering Euclidean distance along the paths in the graph. Nevertheless, their results are roughly in line with ours: if the graph is a tree, then strategy-proof and onto deterministic rules (unanimity is implied) are characterized by so-called extended generalized median voter schemes (cf. Moulin, 1980); for other graphs, there is dictatorship on cycles but if a graph has a leaf then other rules are possible. For earlier work concerning social choice for single-peaked preferences on trees see Hansen and Thisse (1981) and Demange (1982).

Our results show that unanimity and strategy-proofness of probabilistic rules for singlepeaked preferences on graphs imply that these rules are tops-only - they depend only on the peaks of the preferences. In fact, we start out by deriving this result using Theorem 1 in Chatterji and Zeng (2018), see Lemma 2.6. From this lemma we then easily obtain that our rules are uncompromising on trees (cf. Border and Jordan, 1983): if an agent changes its peak, then probabilities assigned to alternatives off the path between the old peak and the new peak remain unaltered (Lemma 3.2). ${ }^{1}$

The literature on strategy-proof probabilistic social choice functions or rules started with the paper of Gibbard (1977), who showed that without restrictions on preferences the conditions of unanimity and strategy-proofness result in random dictatorship. The single-peaked domain restriction (which dates back at least to Black, 1984) allows for other rules, which can be seen as probabilistic extensions of the generalized median rules (Moulin, 1980; Barberà et al, 1993; and others): as already mentioned see Ehlers et al (2002) and Peters et al (2014) for the case with finitely many agents who have single-peaked preferences on the real line or a finite subset of the real line. Dutta et al (2002) show that even under single-peaked preferences, every unanimous and strategy-proof probabilistic rule is a random dictatorship if the dimension is higher than one. ${ }^{2}$ Chatterji et al (2016) show a kind of converse to (among others) our results: a domain has to be single-peaked in order to allow for the existence of unanimous and strategy-proof probabilistic rules satisfying two additional conditions. ${ }^{3}$ See also Chatterji and Massó (2018) for a similar result in the deterministic case. For unanimous and strategy-proof probabilistic rules when preferences are cardinal see the seminal work of Hylland (1980), and further Dutta et al (2007) and Nandeibam (2013).

\footnotetext{
${ }^{1}$ In an earlier version of the paper (Peters et al, 2018) uncompromisingness on trees was derived independently for a smaller set of single-peaked preferences.

${ }^{2}$ In spirit, this result is in line with our result on leafless graphs (Theorem 4.7).

${ }^{3}$ Namely, tops-onliness and a 'compromise' property. Under the assumptions in our paper tops-onliness follows from the other conditions. The 'compromise' property is not necessarily satisfied by a monotonic probabilistic rule.
} 
The paper is organized as follows. After preliminaries in Section 2, including the result that a unanimous and strategy-proof rule is tops-only, we consider the tree case in Section 3 and the leafless graph case in Section 4. Our main and most general result is derived in Section 5. In Section 6 we show that in this context a probabilistic rule on a tree is not necessarily a convex combination of deterministic rules. Section 7 concludes. An appendix presents the proof of Lemma 2.6 on tops-onliness.

\section{Preliminaries}

Let $A$ be a finite set of at least two alternatives and let $N=\{1, \ldots, n\}$ with $n \geq 2$ be a finite set of agents. A complete, reflexive, antisymmetric, and transitive binary relation over $A$ is called a preference. We denote by $\mathbb{L}(A)$ the set of all preferences. A collection of preferences $P_{N}=\left(P_{1}, \ldots, P_{n}\right) \in \mathbb{L}(A)^{N}$ is called a preference profile.

For a preference $P \in \mathbb{L}(A)$ we denote the top alternative by $t(P)$, i.e., $t(P)=a$ if and only if $a P x$ for all $x \in A$. The upper contour set of an alternative $a$ at preference $P$ is the set $U(a, P)=\{x \in A: x P a\}^{4}$

For $P_{N} \in \mathbb{L}(A)^{N}$ and $i \in N, P_{-i} \in \mathbb{L}(A)^{N \backslash\{i\}}$ denotes the restriction of $P_{N}$ to $N \backslash\{i\}$.

\subsection{Single-peaked preferences}

Single-peaked preferences in social choice were already introduced in Black (1948) and Inada (1964). Here, we consider a generalization.

First, we introduce a graph structure on the set of alternatives. A pair $G=(A, E)$, where $E \subseteq\{\{a, b\}: a, b \in A, a \neq b\}$, is an (undirected) graph. The elements of $E$ are called edges. The degree of $a \in A$ is the number $|\{\{x, y\} \in E: a \in\{x, y\}\}|$. A leaf is an alternative with degree one, and $L(G) \subseteq A$ is the set of leafs.

For $a, b \in A$ with $a \neq b$, a path from $a$ to $b$ in $G$ is a sequence of distinct alternatives $a_{1}, \ldots, a_{k}$ such that $a_{1}=a, a_{k}=b$, and $\left\{a_{i}, a_{i+1}\right\} \in E$ for all $i=1, \ldots, k-1$. If it is clear which path is meant, we also denote it by $[a, b]$. In this case, by $(a, b]$ we denote the sequence $a_{2}, \ldots, a_{k}$, and by $(a, b)$ the sequence $a_{2}, \ldots, a_{k-1}$. Whenever it is clear from the context, the notations $[a, b],(a, b]$, and $(a, b)$ will also be used to denote the sets of alternatives (instead of the sequences) that appear in the path. When $a=b$, the notation $[a, b]$ simply denotes the alternative $a, x \in[a, b]$ means $x=a$, and $x \notin[a, b]$ means $x \neq a$.

Throughout this paper we assume that $G$ is connected, i.e., there is a path from $a$ to $b$ for all distinct $a, b \in A$. If this path is unique for all $a, b \in A$, then $G$ is a tree. A spanning tree of $G$ is a tree $T=\left(A, E_{T}\right)$ where $E_{T} \subseteq E$.

For a path $\left[x_{1}, x_{\ell}\right]$ with sequence $x_{1}, \ldots, x_{\ell}$, we write $P=\left[x_{1}, x_{l}\right] \cdots$ to denote a preference $P$ with $x_{1} P x_{2} P \cdots P x_{\ell} P x$ for all $x \in A \backslash\left[x_{1}, x_{\ell}\right]$. Notations like $P=\cdots\left[x_{1}, x_{\ell}\right] \cdots$

and combinations of these have similar meanings. Also, brackets are sometimes left out if confusion is unlikely.

Definition 2.1. A preference $P$ is single-peaked if there is a spanning tree $T$ of $G$ such that for all distinct $x, y \in A$ with $t(P) \neq y$,

$$
x \in[t(P), y] \Longrightarrow x P y,
$$

\footnotetext{
${ }^{4}$ Observe that $a \in U(a, P)$ by reflexivity.
} 
where $[t(P), y]$ is the path from $t(P)$ to $y$ in $T$. By $\mathcal{S} \subseteq \mathbb{L}(A)$ we denote the set of all single-peaked preferences. For a single-peaked preference, the top alternative is also called the peak.

\subsection{Probabilistic rules}

By $\triangle A$, we denote the set of all probability distributions on $A$. A probabilistic rule (PR) is a function $\varphi: \mathcal{S}^{N} \rightarrow \triangle A$. For $B \subseteq A$ and $P_{N} \in \mathcal{S}^{N}$, we write $\varphi_{B}\left(P_{N}\right)=\sum_{a \in B} \varphi_{a}\left(P_{N}\right)$, where $\varphi_{a}\left(P_{N}\right)$ is the probability of $a$ at $\varphi\left(P_{N}\right)$.

We proceed by defining the main properties of PRs that are of interest in this paper. The first property needs no explanation.

Definition 2.2. A PR $\varphi$ is unanimous if $\varphi_{a}\left(P_{N}\right)=1$ for all $a \in A$ and all $P_{N} \in \mathcal{S}^{N}$ with $t\left(P_{i}\right)=a$ for all $i \in N$.

The second property is the strategy-proofness condition introduced in Gibbard (1977). It says that reporting a preference different from the sincere (true) one cannot increase the probability on any sincere upper contour set; in other words, the probability distribution over the alternatives induced by reporting truthfully stochastically dominates any probability distribution induced by reporting differently.

Definition 2.3. A $\operatorname{PR} \varphi$ is strategy-proof if for all $i \in N$, all $P_{N} \in \mathcal{S}^{N}$, all $P_{i}^{\prime} \in \mathcal{S}$, and all $x \in A$,

$$
\varphi_{U\left(x, P_{i}\right)}\left(P_{i}, P_{-i}\right) \geq \varphi_{U\left(x, P_{i}\right)}\left(P_{i}^{\prime}, P_{-i}\right) .
$$

It is not hard to see that under strategy-proofness the unanimity condition could be weakened to requiring $\varphi_{a}\left(P_{N}\right)=1$ for all $a \in A$ and all $P_{N} \in \mathcal{S}^{N}$ with $P_{i}=P_{j}$ and $t\left(P_{i}\right)=a$ for all $i, j \in N$. For later reference we also include the following observation.

Remark 2.4. Let $L, L^{\prime} \in \triangle A$ and let $P \in \mathbb{L}(A)$. Suppose $L_{U(x, P)}=L_{U(x, P)}^{\prime}$ for all $x \in A$, where $L_{U(x, P)}$ denotes the total probability on the upper contour set $U(x, P)$. Then $L=L^{\prime}$.

Two profiles $P_{N}, P_{N}^{\prime} \in \mathcal{S}^{N}$ are tops-equivalent if $t\left(P_{i}\right)=t\left(P_{i}^{\prime}\right)$ for all $i \in N$. The following condition again needs no explanation.

Definition 2.5. A PR $\varphi$ is tops-only if $\varphi\left(P_{N}\right)=\varphi\left(P_{N}^{\prime}\right)$ for all tops-equivalent $P_{N}, P_{N}^{\prime} \in \mathcal{S}^{N}$.

In our model, unanimity and strategy-proofness of a PR imply tops-onliness. This can be proved by using the main result in Chatterji and Zeng (2018), as we show in the Appendix.

Lemma 2.6. Let $G=(A, E)$ be a connected graph and let $P R \varphi$ on $\mathcal{S}^{N}$ be unanimous and strategy-proof. Then $\varphi$ is tops-only.

Proof. See Appendix A. 


\section{Trees}

Throughout this section the graph $G=(A, E)$ is a tree. We will characterize all unanimous and strategy-proof probabilistic rules for this case. To this end the following property will be very useful.

Definition 3.1. Let $G=(A, E)$ be a tree. $\mathrm{A} \operatorname{PR} \varphi: \mathcal{S}^{N} \rightarrow \triangle A$ is uncompromising if $\varphi_{d}\left(P_{N}\right)=\varphi_{d}\left(P_{i}^{\prime}, P_{-i}\right)$ for all $i \in N$, all $P_{N} \in \mathcal{S}^{N}$, all $P_{i}^{\prime} \in \mathcal{S}$ and all $d \in A$ such that $d \notin\left[t\left(P_{i}\right), t\left(P_{i}^{\prime}\right)\right]$.

This property, which was first introduced by Border and Jordan (1983) for deterministic rules, says that if an agent changes its preference then the probabilities of alternatives which are not on the path between the former and the new peak of that agent, do not change. Uncompromisingness is closely related to strategy-proofness but often easier to work with. Clearly, an uncompromising PR is tops-only. The latter property also follows from Lemma 2.6. In the following lemma we show that, by using tops-onliness, uncompromisingness can easily be derived from unanimity and strategy-proofness.

Lemma 3.2. Let $G=(A, E)$ be a tree and let $\varphi: \mathcal{S}^{N} \rightarrow \triangle A$ be a unanimous and strategyproof $P R$. Then $\varphi$ is uncompromising.

Proof. Let $P_{N}, P_{N}^{\prime} \in \mathcal{S}^{N}$ and $i \in N$ such that $P_{-i}=P_{-i}^{\prime}$. In order to prove that $\varphi_{x}\left(P_{N}\right)=$ $\varphi_{x}\left(P_{N}^{\prime}\right)$ for all $x \notin\left[t\left(P_{i}\right), t\left(P_{i}^{\prime}\right)\right]$, it is without loss of generality to assume $\left\{t\left(P_{i}\right), t\left(P_{i}^{\prime}\right)\right\} \in E$. Then, by tops-onliness (Lemma 2.6), we may assume that $P_{i}=t\left(P_{i}\right) t\left(P_{i}^{\prime}\right) \cdots$ and $P_{i}^{\prime}=$ $t\left(P_{i}^{\prime}\right) t\left(P_{i}\right) \cdots$ such that $z P_{i} z^{\prime} \Leftrightarrow z P_{i}^{\prime} z^{\prime}$ for all $z, z^{\prime} \in A \backslash\left\{t\left(P_{i}\right), t\left(P_{i}^{\prime}\right)\right\}$. Now the lemma follows directly from strategy-proofness.

In what follows we show that a unanimous and strategy-proof PR is completely determined by its values for profiles where the peaks of the agents are located on the leafs of the tree. Therefore, we introduce the following definition.

Definition 3.3. A leaf assignment is a function $\mu: N \rightarrow L(G)$. The set of all leaf assignments is denoted by $\mathcal{M}$. For $a \in A$ and $P_{N} \in \mathcal{S}^{N}$, a leaf assignment $\mu$ is monotonic with respect to $\left(a, P_{N}\right)$ if for all $i \in N$ and $b \in L(G), \mu(i)=b$ implies $t\left(P_{i}\right) \in[a, b]$. The set of leaf assignments that are monotonic with respect to $\left(a, P_{N}\right)$ is denoted by $\mathcal{M}\left(a, P_{N}\right)$.

Thus, a leaf assignment assigns to each agent a leaf of the tree. If a leaf assignment is monotonic with respect to an alternative $a$ and a preference profile, then the peak of each agent must be on the path from $a$ to the leaf which is assigned to that agent. If, in

particular, this leaf is equal to $a$, then that agent's peak is also $a$. Clearly, if $P_{N}, P_{N}^{\prime} \in \mathcal{S}^{N}$ are tops-equivalent, then $\mathcal{M}\left(a, P_{N}\right)=\mathcal{M}\left(a, P_{N}^{\prime}\right)$. The following example illustrates Definition 3.3 .

Example 3.4. Consider the following tree.

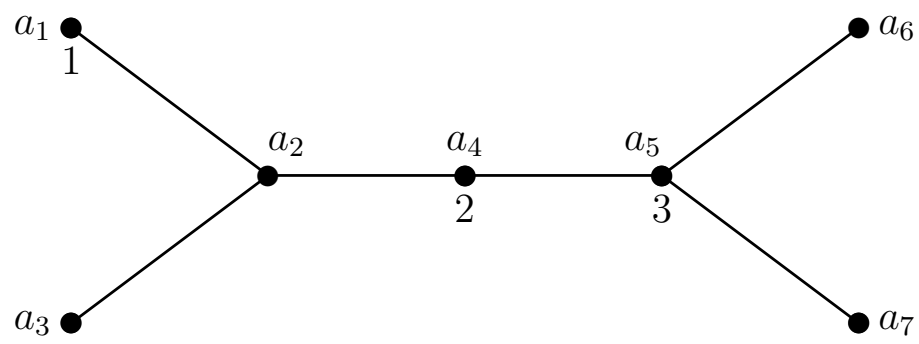


Let $N=\{1,2,3\}$, and let $P_{N}$ be a profile with $\left(t\left(P_{1}\right), t\left(P_{2}\right), t\left(P_{3}\right)\right)=\left(a_{1}, a_{4}, a_{5}\right)$, as illustrated in the picture. Then

$$
\begin{aligned}
& \mu \in \mathcal{M}\left(a_{1}, P_{N}\right) \Leftrightarrow \mu(1) \in\left\{a_{1}, a_{3}, a_{6}, a_{7}\right\}, \mu(2), \mu(3) \in\left\{a_{6}, a_{7}\right\} \\
& \mu \in \mathcal{M}\left(a_{2}, P_{N}\right) \Leftrightarrow \mu(1)=a_{1}, \mu(2), \mu(3) \in\left\{a_{6}, a_{7}\right\} \\
& \mu \in \mathcal{M}\left(a_{3}, P_{N}\right) \Leftrightarrow \mu(1)=a_{1}, \mu(2), \mu(3) \in\left\{a_{6}, a_{7}\right\} \\
& \mu \in \mathcal{M}\left(a_{4}, P_{N}\right) \Leftrightarrow \mu(1)=a_{1}, \mu(2) \in\left\{a_{1}, a_{3}, a_{6}, a_{7}\right\}, \mu(3) \in\left\{a_{6}, a_{7}\right\} \\
& \mu \in \mathcal{M}\left(a_{5}, P_{N}\right) \Leftrightarrow \mu(1)=a_{1}, \mu(2) \in\left\{a_{1}, a_{3}\right\}, \mu(3) \in\left\{a_{1}, a_{3}, a_{6}, a_{7}\right\} \\
& \mu \in \mathcal{M}\left(a_{6}, P_{N}\right) \Leftrightarrow \mu(1)=a_{1}, \mu(2) \in\left\{a_{1}, a_{3}\right\}, \mu(3) \in\left\{a_{1}, a_{3}, a_{7}\right\} \\
& \mu \in \mathcal{M}\left(a_{7}, P_{N}\right) \Leftrightarrow \mu(1)=a_{1}, \mu(2) \in\left\{a_{1}, a_{3}\right\}, \mu(3) \in\left\{a_{1}, a_{3}, a_{6}\right\}
\end{aligned}
$$

With each $\mu \in \mathcal{M}$ we associate a probability distribution $\beta_{\mu}$ over $A$.

Definition 3.5. A collection of probability distributions $\left(\beta_{\mu}\right)_{\mu \in \mathcal{M}}$ over $A$ is called monotonic if

(i) for every $b \in L(G)$ and $\mu \in \mathcal{M}$, if $\mu(i)=b$ for all $i \in N$, then $\beta_{\mu}(b)=1$,

(ii) for all $\mu, \hat{\mu} \in \mathcal{M}$ and $i \in N$ such that $\mu(j)=\hat{\mu}(j)$ for all $j \in N \backslash\{i\}$,

(a) $\beta_{\hat{\mu}}([c, \hat{\mu}(i)]) \geq \beta_{\mu}([c, \hat{\mu}(i)])$ for all $c \in[\mu(i), \hat{\mu}(i)]$,

(b) $\beta_{\mu}(c)=\beta_{\hat{\mu}}(c)$ for all $c \in A \backslash[\mu(i), \hat{\mu}(i)]$.

Part (i) in this definition says that if all agents are assigned to the same leaf then that leaf obtains probability one. Part (ii) says that if an agent $i$ moves from one leaf (at $\mu$ ) to another (at $\hat{\mu}$ ), then probability increases along the path from the former to the latter leaf (part (a)), whereas off this path nothing changes (part (b)). Clearly, the conditions (i), (ii)(a), and (ii)(b), are related to the conditions of unanimity, strategy-proofness, and uncompromisingness of a PR.

The following example illustrates the notion of monotonic probability distributions.

Example 3.6. Consider again the tree of Example 3.4 but now assume that there are three agents, $N=\{1,2,3\}$. For convenience we replicate the tree.

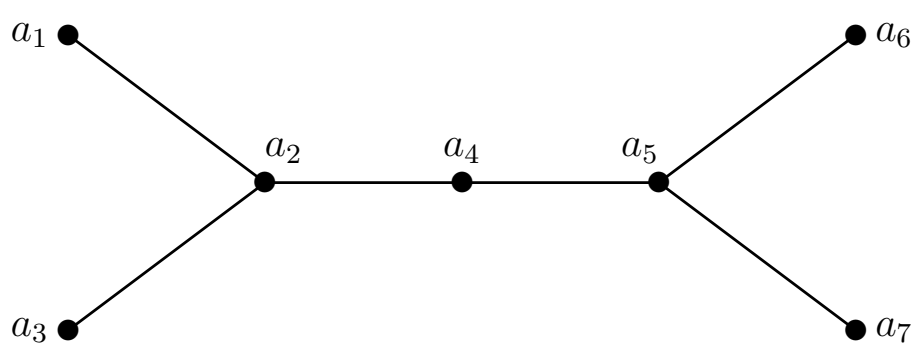

Consider the probability distributions $\left(\beta_{\mu}\right)_{\mu \in \mathcal{M}}$ in the table below. For simplicity, we assume that the collection $\left(\beta_{\mu}\right)_{\mu \in \mathcal{M}}$ is 'anonymous', which means that the probabilities depend only on the numbers of agents on the leafs. The $\mu$-assignments are to the leafs $a_{1}, a_{3}, a_{6}$, and $a_{7}$ consecutively. The probabilities (the numbers in the table divided by 10) are those assigned to $a_{1}, \ldots, a_{7}$, consecutively. It is left to the reader to verify that $\left(\beta_{\mu}\right)_{\mu \in \mathcal{M}}$ in this table satisfies monotonicity. 


\begin{tabular}{c|c||c|c}
$\mu$ & $\beta_{\mu}$ & $\mu$ & $\beta_{\mu}$ \\
\hline$(3,0,0,0)$ & $(10,0,0,0,0,0,0)$ & $(1,0,2,0)$ & $(1,3,0,2,2,2,0)$ \\
$(0,3,0,0)$ & $(0,0,10,0,0,0,0)$ & $(0,1,2,0)$ & $(0,2,3,2,1,2,0)$ \\
$(0,0,3,0)$ & $(0,0,0,0,0,10,0)$ & $(0,0,2,1)$ & $(0,0,0,0,7,2,1)$ \\
$(0,0,0,3)$ & $(0,0,0,0,0,0,10)$ & $(1,0,0,2)$ & $(1,3,0,2,2,0,2)$ \\
$(2,1,0,0)$ & $(4,3,3,0,0,0,0)$ & $(0,1,0,2)$ & $(0,2,3,2,1,0,2)$ \\
$(2,0,1,0)$ & $(4,2,0,2,1,1,0)$ & $(0,0,1,2)$ & $(0,0,0,0,7,1,2)$ \\
$(2,0,0,1)$ & $(4,2,0,2,1,0,1)$ & $(1,1,1,0)$ & $(1,2,3,2,1,1,0)$ \\
$(1,2,0,0)$ & $(1,5,4,0,0,0,0)$ & $(1,1,0,1)$ & $(1,2,3,2,1,0,1)$ \\
$(0,2,1,0)$ & $(0,2,4,2,1,1,0)$ & $(1,0,1,1)$ & $(1,3,0,3,1,1,1)$ \\
$(0,2,0,1)$ & $(0,2,4,2,1,0,1)$ & $(0,1,1,1)$ & $(0,3,1,3,1,1,1)$
\end{tabular}

Below we associate a PR with each monotonic collection of probability distributions. As a preparation we need the following lemma.

Lemma 3.7. Let $\left(\beta_{\mu}\right)_{\mu \in \mathcal{M}}$ be a monotonic collection of probability distributions. Let $a \in A$, $b, c \in L(G), P_{N} \in \mathcal{S}^{N}$, and $\mu_{b}, \hat{\mu}_{b}, \mu_{c}, \hat{\mu}_{c} \in \mathcal{M}\left(a, P_{N}\right)$ such that for each $x \in\{b, c\}$ and all $i \in N, \mu_{x}(i)=x$ if and only if $t\left(P_{i}\right) \in[a, x]$ and $\hat{\mu}_{x}(i)=x$ if and only if $t\left(P_{i}\right) \in(a, x]$. Then

$$
\beta_{\mu_{b}}([a, b])-\beta_{\hat{\mu}_{b}}((a, b])=\beta_{\mu_{c}}([a, c])-\beta_{\hat{\mu}_{c}}((a, c]) \geq 0 .
$$

Proof. First, we argue that the left-hand side of (1) does not depend on the particular choice of $\hat{\mu}_{b}$. An analogous argument holds for the right-hand side. Take $\hat{\mu}_{b}^{\prime} \in \mathcal{M}\left(a, P_{N}\right)$ such that for all $i \in N, \hat{\mu}_{b}^{\prime}(i)=b$ if and only if $t\left(P_{i}\right) \in(a, b]$. Then, by condition (ii)(b) in Definition 3.5 we have

$$
\beta_{\hat{\mu}_{b}}((a, b])=\beta_{\hat{\mu}_{b}^{\prime}}((a, b]) .
$$

It is sufficient to prove the lemma for the case where $a \in[b, c]$. Otherwise, there is a $d \in L(G)$ such that both $a \in[d, b]$ and $a \in[d, c]$. Then, if we show (1) for the pairs of leafs $b, d$ and $c, d,(1)$ follows for the pair $b, c$ by combining the two equations. Thus, we assume $a \in[b, c]$. For the equality in (1), it is in view of (2) sufficient to show that

$$
\beta_{\mu_{b}}([a, b])-\beta_{\mu_{c}}((a, b])=\beta_{\mu_{c}}([a, c])-\beta_{\mu_{b}}((a, c]) .
$$

By condition (ii)(b) in Definition 3.5 we have

$$
\beta_{\mu_{b}}[b, c]=\beta_{\mu_{c}}[b, c]
$$

Therefore,

$$
\begin{aligned}
\beta_{\mu_{b}}([a, b])-\beta_{\mu_{c}}((a, b]) & =\beta_{\mu_{b}}([b, c])-\beta_{\mu_{b}}((a, c])-\beta_{\mu_{c}}((a, b]) \\
& =\beta_{\mu_{c}}([b, c])-\beta_{\mu_{c}}((a, b])-\beta_{\mu_{b}}((a, c]) \\
& =\beta_{\mu_{c}}([a, c])-\beta_{\mu_{b}}((a, c])
\end{aligned}
$$

where the second equality follows from (3).

Finally, by condition (ii)(a) in Definition 3.5 we have

$$
\beta_{\hat{\mu}_{b}}((a, b]) \leq \beta_{\mu_{b}}((a, b])
$$

which implies the nonnegativity of the expressions in (1) and completes the proof of the lemma. 
Let $B=\left(\beta_{\mu}\right)_{\mu \in \mathcal{M}}$ be a monotonic collection of probability distributions over $A$. With $B$ we associate a map $\varphi^{B}: \mathcal{S}^{N} \rightarrow \triangle A$ by defining, for each $a \in A$ and $P_{N} \in \mathcal{S}_{N}$,

$$
\varphi_{a}^{B}\left(P_{N}\right)=\beta_{\mu_{b}}([a, b])-\beta_{\hat{\mu}_{b}}((a, b])
$$

for some $b \in L(G)$ and $\mu_{b}, \hat{\mu}_{b} \in \mathcal{M}\left(a, P_{N}\right)$ such that $\mu_{b}(i)=b$ if and only if $t\left(P_{i}\right) \in[a, b]$ and $\hat{\mu}_{b}(i)=b$ if and only if $t\left(P_{i}\right) \in(a, b]$. In words, the probability assigned by $\varphi^{B}$ to alternative $a$ if the preference profile is $P_{N}$, is determined as follows. Take an arbitrary leaf $b$, take a leaf assignment where all agents with peaks on the path $[a, b]$ are assigned to $b$, and compute the sum of the associated probabilities assigned to alternatives on the path $[a, b]$. Next, take a leaf assignment where all agents with peaks on the path $(a, b]$ (but not those with peak at $a$ ) are assigned to $b$, and compute the sum of the associated probabilities assigned to alternatives on the path $(a, b]$. The difference between these numbers is the probability assigned to $a$.

Note that by Lemma 3.7, $\varphi^{B}$ is well-defined: it does not depend on the particular choice of $b, \mu_{b}$, or $\hat{\mu}_{b}$. Moreover we have:

Lemma 3.8. $\varphi^{B}$ defined by (4) is a PR.

Proof. By Lemma 3.7, $\varphi_{a}^{B}\left(P_{N}\right) \geq 0$ for every $a \in A$ and $P_{N} \in \mathcal{S}^{N}$. We still have to prove that $\sum_{a \in A} \varphi_{a}^{B}\left(P_{N}\right)=1$ for every $P_{N} \in \mathcal{S}^{N}$.

Let $a \in A, b \in L(G)$, and let $\mu \in \mathcal{M}\left(a, P_{N}\right)$ such that $\mu(i)=b$ if and only if $t\left(P_{i}\right) \in[a, b]$, for all $i \in N$. We claim that $\varphi_{[a, b]}^{B}\left(P_{N}\right)=\beta_{\mu}([a, b])$. To show this, write $[a, b]=\left(a_{1}, \ldots, a_{k}\right)$, where $a=a_{1}$ and $b=a_{k}$. For every $j=1, \ldots, k$ let $\mu_{j}, \hat{\mu}_{j} \in \mathcal{M}\left(a_{j}, P_{N}\right)$ such that for all $i \in N$ we have $\mu(j)=b \Leftrightarrow t\left(P_{i}\right) \in\left[a_{j}, b\right]$ and $\hat{\mu}(j)=b \Leftrightarrow t\left(P_{i}\right) \in\left(a_{j}, b\right]$. Then

$$
\begin{aligned}
\varphi_{[a, b]}^{B}\left(P_{N}\right)= & \beta_{\mu_{1}}\left(\left[a_{1}, b\right]\right)-\beta_{\hat{\mu}_{1}}\left(\left(a_{1}, b\right]\right) \\
& +\beta_{\mu_{2}}\left(\left[a_{2}, b\right]\right)-\beta_{\hat{\mu}_{2}}\left(\left(a_{2}, b\right]\right) \\
& +\beta_{\mu_{3}}\left(\left[a_{3}, b\right]\right)-\beta_{\hat{\mu}_{3}}\left(\left(a_{3}, b\right]\right) \\
& \vdots \\
& +\beta_{\mu_{k}}(\{b\})-\beta_{\hat{\mu}_{k}}(\emptyset) \\
= & \beta_{\mu_{1}}\left(\left[a_{1}, b\right]\right) \\
= & \beta_{\mu}([a, b])
\end{aligned}
$$

where the before last equality follows since $\beta_{\hat{\mu}_{j}}\left(\left(a_{j}, b\right]\right)=\beta_{\hat{\mu}_{j}}\left(\left[a_{j+1}, b\right]\right)=\beta_{\mu_{j+1}}\left(\left[a_{j+1}, b\right]\right)$ for every $j=1, \ldots, k-1$ by condition (ii)(b) in Definition 3.5, and also the last equality follows from this condition.

Now let $P_{N} \in \mathcal{S}^{N}$ be arbitrary. We partition $A$ into subsets $A^{1}, \ldots, A^{k}$, such that each $A^{\ell}$ is of the form $A^{\ell}=\left[a^{\ell}, \ldots, b^{\ell}\right]$ for some $a^{\ell} \in A$ and $b^{\ell} \in L(G)$ (possibly $a^{\ell}=b^{\ell}$ ). We define the leaf assignment $\mu$ as follows: (i) for each $\ell=1, \ldots, k, \mu^{-1}\left(b^{\ell}\right)=\left\{i \in N: t\left(P_{i}\right) \in A^{\ell}\right\}$, and (ii) for each $b \in L(G) \backslash\left\{b^{1}, \ldots, b^{k}\right\}, \mu^{-1}(b)=\emptyset$ (case (ii) occurs if $b=a^{\ell}$ for some $\ell$ ). Then by condition (ii)(b) in Definition 3.5, for every $\ell=1, \ldots, k$ there is a $\mu_{\ell} \in \mathcal{M}\left(a^{\ell}, P_{N}\right)$ such that $\beta_{\mu_{\ell}}\left(A^{\ell}\right)=\beta_{\mu}\left(A^{\ell}\right)$. Hence, by the previous part of the proof, $\sum_{a \in A} \varphi_{a}^{B}\left(P_{N}\right)=$ $\sum_{\ell=1}^{k} \beta_{\mu_{\ell}}\left(A^{\ell}\right)=\sum_{\ell=1}^{k} \beta_{\mu}\left(A^{\ell}\right)=\beta_{\mu}(A)=1$.

Definition 3.9. A PR $\varphi$ is monotonic if there is a monotonic collection of probability distributions $B=\left(\beta_{\mu}\right)_{\mu \in \mathcal{M}}$ such that $\varphi=\varphi^{B}$. 
An example of a monotonic PR is the following.

Example 3.10. Consider the tree of Example 3.6. Let $N=\{1,2,3\}$. Let $\varphi$ be the monotonic (anonymous, i.e., invariant under any permutation of the agents) PR with respect to $\left(\beta_{\mu}\right)_{\mu \in \mathcal{M}}$ as in Example 3.6. Consider the profile $P_{N}$ with $\left(t\left(P_{1}\right), t\left(P_{2}\right), t\left(P_{3}\right)\right)=\left(a_{1}, a_{4}, a_{5}\right)$ as in Example 3.4. We take the fixed leaf $a_{1}$ for the computations in the following table, which provides the outcome of $\varphi$ at $P_{N}$.

\begin{tabular}{c|c|c|c}
$a$ & $b$ & $\beta_{\mu}([a, b])-\beta_{\mu^{\prime}}((a, b])$ & $\varphi_{a}\left(P_{N}\right)$ \\
\hline$a_{1}$ & $a_{1}$ & $\beta_{(1,0,2,0)}\left(\left[a_{1}, a_{1}\right]\right)-\beta_{(0,0,3,0)}\left(\left(a_{1}, a_{1}\right]\right)$ & .1 \\
$a_{2}$ & $a_{1}$ & $\beta_{(1,0,2,0)}\left(\left[a_{2}, a_{1}\right]\right)-\beta_{(1,0,2,0)}\left(\left(a_{2}, a_{1}\right]\right)$ & .3 \\
$a_{3}$ & $a_{1}$ & $\beta_{(1,0,2,0)}\left(\left[a_{3}, a_{1}\right]\right)-\beta_{(1,0,2,0)}\left(\left(a_{3}, a_{1}\right]\right)$ & 0 \\
$a_{4}$ & $a_{1}$ & $\beta_{(2,0,1,0)}\left(\left[a_{4}, a_{1}\right]\right)-\beta_{(1,0,2,0)}\left(\left(a_{4}, a_{1}\right]\right)$ & .4 \\
$a_{5}$ & $a_{1}$ & $\beta_{(3,0,0,0)}\left(\left[a_{5}, a_{1}\right]\right)-\beta_{(2,0,1,0)}\left(\left(a_{5}, a_{1}\right]\right)$ & .2 \\
$a_{6}$ & $a_{1}$ & $\beta_{(3,0,0,0)}\left(\left[a_{6}, a_{1}\right]\right)-\beta_{(3,0,0,0)}\left(\left(a_{6}, a_{1}\right]\right)$ & 0 \\
$a_{7}$ & $a_{1}$ & $\beta_{(3,0,0,0)}\left(\left[a_{7}, a_{1}\right]\right)-\beta_{(3,0,0,0)}\left(\left(a_{7}, a_{1}\right]\right)$ & 0
\end{tabular}

Our main result will be that these monotonic PRs are exactly the unanimous and strategy-proof PRs for single-peaked preferences on trees.

Lemma 3.11. Let $B=\left(\beta_{\mu}\right)_{\mu \in \mathcal{M}}$ be a monotonic collection of probability distributions over A. Then $\varphi^{B}$ is unanimous and strategy-proof.

Proof. In this proof we write $\varphi$ instead of $\varphi^{B}$. Unanimity follows directly from the definition of $\varphi$.

We next argue that $\varphi$ is uncompromising. Let $P_{N} \in \mathcal{S}^{N}, i \in N, P_{i}^{\prime} \in \mathcal{S}$, and $d \in$ $A \backslash\left[t\left(P_{i}\right), t\left(P_{i}^{\prime}\right)\right]$. Take $b \in L(G)$ such that $[d, b] \cap\left[t\left(P_{i}\right), t\left(P_{i}^{\prime}\right)\right]=\emptyset$. Then, by definition of $\varphi$, in particular $(4)$, we obtain $\varphi_{d}\left(P_{N}\right)=\varphi_{d}\left(P_{-i}, P_{i}^{\prime}\right)$. This shows that $\varphi$ is uncompromising.

In order to prove strategy-proofness, assume for contradiction that there exists $i \in N$, $P_{N} \in \mathcal{S}^{N}$, and $P_{i}^{\prime} \in \mathcal{S}$ such that $\varphi_{U\left(c, P_{i}\right)}\left(P_{N}\right)<\varphi_{U\left(c, P_{i}\right)}\left(P_{i}^{\prime}, P_{-i}\right)$ for some $c \in A$. Since $\varphi$ is uncompromising and thus tops-only, we may assume without loss of generality that $P_{i}=\left[t\left(P_{i}\right), \ldots, t\left(P_{i}^{\prime}\right)\right] \cdots$ and $P_{i}^{\prime}=\left[t\left(P_{i}^{\prime}\right), \ldots, t\left(P_{i}\right)\right] \cdots$, and by uncompromisingness we also have $\varphi_{z}\left(P_{N}\right)=\varphi_{z}\left(P_{i}^{\prime}, P_{-i}\right)$ for all $z \notin\left[t\left(P_{i}\right), t\left(P_{i}^{\prime}\right)\right]$. Therefore, we may assume without loss of generality that $c \in\left[t\left(P_{i}\right), t\left(P_{i}^{\prime}\right)\right)$ and

$$
\varphi_{\left[t\left(P_{i}\right), c\right]}\left(P_{N}\right)<\varphi_{\left[t\left(P_{i}\right), c\right]}\left(P_{i}^{\prime}, P_{-i}\right) .
$$

Let $d$ appear just after $c$ on the path $\left[t\left(P_{i}\right), t\left(P_{i}^{\prime}\right)\right]$. Let $P^{c} \in \mathcal{S}$ with $t\left(P^{c}\right)=c$ and $P^{d} \in \mathcal{S}$ with $t(P)=d$. By uncompromisingness, $\varphi_{\left[t\left(P_{i}\right), c\right]}\left(P_{N}\right)=\varphi_{\left[t\left(P_{i}\right), c\right]}\left(P^{c}, P_{-i}\right)$ and $\varphi_{\left[t\left(P_{i}\right), c\right]}\left(P^{d}, P_{-i}\right)=\varphi_{\left[t\left(P_{i}\right), c\right]}\left(P_{i}^{\prime}, P_{-i}\right)$. By $(5)$, this yields $\varphi_{\left[t\left(P_{i}\right), c\right]}\left(P^{c}, P_{-i}\right)<\varphi_{\left[t\left(P_{i}\right), c\right]}\left(P^{d}, P_{-i}\right)$. Since by uncompromisingness $\varphi_{z}\left(P^{c}, P_{-i}\right)=\varphi_{z}\left(P^{d}, P_{-i}\right)$ for all $z \notin\{c, d\}$, this implies

$$
\varphi_{c}\left(P^{c}, P_{-i}\right)<\varphi_{c}\left(P^{d}, P_{-i}\right) \text {. }
$$

Now take $b, b^{\prime} \in L(G)$ such that $\{c, d\} \subseteq\left[b, b^{\prime}\right]$ and $d \notin[b, c]$. By (4),

$$
\varphi_{c}\left(P^{c}, P_{-i}\right)=\beta_{\mu_{b}}([c, b])-\beta_{\hat{\mu}_{b}}((c, b])
$$

where $\mu_{b}, \hat{\mu}_{b} \in \mathcal{M}\left(c,\left(P^{c}, P_{-i}\right)\right)$ are such that $\mu_{b}(j)=b$ if and only if $t\left(P_{j}\right) \in[c, b]$ and $\hat{\mu}_{b}(j)=b$ if and only if $t\left(P_{j}\right) \in(c, b]$ for all $j \in N$. Let $\mu_{b}^{\prime}$ be such that $\mu_{b}^{\prime}(j)=\mu(j)$ for all $j \in N \backslash\{i\}$ and $\mu_{b}^{\prime}(i)=b^{\prime}$; and let $\hat{\mu}_{b}^{\prime}=\hat{\mu}_{b}$. Note that $\mu_{b}^{\prime}, \hat{\mu}_{b}^{\prime} \in \mathcal{M}\left(c,\left(P^{d}, P_{-i}\right)\right)$. Also, 
writing $\hat{P}_{N}=\left(P^{d}, P_{-i}\right)$, we have $\mu_{b}^{\prime}(j)=b$ if and only if $t\left(\hat{P}_{j}\right) \in[c, b]$ and $\hat{\mu}_{b}^{\prime}(j)=b$ if and only if $t\left(\hat{P}_{j}\right) \in(c, b]$ for all $j \in N$. Therefore, by (4),

$$
\varphi_{c}\left(P^{d}, P_{-i}\right)=\beta_{\mu_{b}^{\prime}}([c, b])-\beta_{\hat{\mu}_{b}^{\prime}}((c, b]) .
$$

By (6), (7), (8), and the fact that $\hat{\mu}_{b}^{\prime}=\hat{\mu}_{b}$, we obtain

$$
\beta_{\mu_{b}}([c, b])<\beta_{\mu_{b}^{\prime}}([c, b]) .
$$

However, as (i) $\mu_{b}^{-1}(\hat{b})=\mu_{b}^{\prime-1}(\hat{b})$ for all $\hat{b} \in L(G) \backslash\left\{b, b^{\prime}\right\}$ and (ii) $\mu_{b}^{\prime-1}(b) \subseteq \mu_{b}^{-1}(b)$, this contradicts condition (ii)(a) in Definition 3.5.

Next we show the converse of Lemma 3.11.

Lemma 3.12. Let $\varphi$ be a unanimous and strategy-proof $P R$. Then there is a monotonic collection of probability distributions $B=\left(\beta_{\mu}\right)_{\mu \in \mathcal{M}}$ such that $\varphi=\varphi^{B}$.

Proof. By Lemma 3.2, $\varphi$ is uncompromising. For every $\mu \in \mathcal{M}$ define $\beta_{\mu}=\varphi\left(P_{N}\right)$, where $P_{N} \in \mathcal{S}^{N}$ satisfies $t\left(P_{i}\right)=\mu(i)$ for all $i \in N$.

We first show that $B=\left(\beta_{\mu}\right)_{\mu \in \mathcal{M}}$ thus defined, is a monotonic collection. Clearly, since $\varphi$ is unanimous, condition (i) in Definition 3.5 is satisfied. For condition (ii), let $\mu, \hat{\mu} \in \mathcal{M}$ and $i \in N$ such that $\mu(j)=\hat{\mu}(j)$ for all $j \in N \backslash\{i\}$ and let $P_{N}, \hat{P}_{N}$ such that $t\left(P_{k}\right)=$ $\mu(k)$ and $t\left(\hat{P}_{k}\right)=\hat{\mu}(k)$ for all $k \in N$. Since $\varphi$ is uncompromising, $\varphi_{c}\left(P_{N}\right)=\varphi_{c}\left(\hat{P}_{N}\right)$ for all $c \notin\left[t\left(P_{i}\right), t\left(\hat{P}_{i}\right)\right]$, hence $\beta_{\mu}(c)=\beta_{\hat{\mu}}(c)$ for all $c \notin[\mu(i), \hat{\mu}(i)]$, i.e., condition (ii)(b) is satisfied. Moreover, by strategy-proofness of $\varphi$ we have for all $c \in\left[t\left(P_{i}\right), t\left(\hat{P}_{i}\right)\right]$ that $\varphi_{U\left(c, \hat{P}_{i}\right)}\left(\hat{P}_{N}\right) \geq \varphi_{U\left(c, \hat{P}_{i}\right)}\left(P_{N}\right)$. Since $\varphi_{z}\left(P_{N}\right)=\varphi_{z}\left(\hat{P}_{N}\right)$ for all $z \notin\left[t\left(P_{i}\right), t\left(\hat{P}_{i}\right)\right]$, this implies $\varphi_{\left[c, \hat{P}_{i}\right]}\left(\hat{P}_{N}\right) \geq \varphi_{\left[c, \hat{P}_{i}\right]}\left(P_{N}\right)$, and therefore $\beta_{\hat{\mu}}([c, \hat{\mu}(i)]) \geq \beta_{\mu}([c, \hat{\mu}(i)])$ for all $c \in[\mu(i), \hat{\mu}(i)]$. This proves condition (ii)(a).

Finally, we show that $\varphi=\varphi^{B}$. Let $P_{N} \in \mathcal{S}^{N}$ and $a \in A$. Let $\mu^{\prime}, \mu^{\prime \prime} \in \mathcal{M}\left(a, P_{N}\right)$ and $b \in L(G)$ such that, for all $i \in N, \mu^{\prime}(i)=b$ if and only if $t\left(P_{i}\right) \in[a, b]$ and $\mu^{\prime \prime}(i)=b$ if and only if $t\left(P_{i}\right) \in(a, b]$. Also, let $P_{N}^{\prime} \in \mathcal{S}^{N}$ such that $t\left(P_{i}^{\prime}\right)=\mu^{\prime}(i)$ for all $i \in N$ and $P_{N}^{\prime \prime} \in \mathcal{S}^{N}$ such that $t\left(P_{i}^{\prime \prime}\right)=\mu^{\prime \prime}(i)$ for all $i \in N$. Then

$$
\begin{aligned}
\varphi_{a}^{B}\left(P^{N}\right) & =\beta_{\mu^{\prime}}([a, b])-\beta_{\mu^{\prime \prime}}((a, b]) \\
& =\varphi_{[a, b]}\left(P_{N}^{\prime}\right)-\varphi_{(a, b]}\left(P_{N}^{\prime \prime}\right) \\
& =\varphi_{a}\left(P_{N}\right)
\end{aligned}
$$

where the last equality follows by uncompromisingness of $\varphi$. We conclude that $\varphi=\varphi^{B}$.

Lemmas 3.11 and 3.12 now imply the main result of this section.

Theorem 3.13. Let $G=(A, E)$ be a tree. Then a $P R \varphi$ on $\mathcal{S}^{N}$ is unanimous and strategyproof if and only if it is monotonic.

Theorem 3.13 implies a characterization of deterministic unanimous and strategy-proof rules on tree as a corollary. See Section 6, where we show in particular that the probabilistic rules with these properties are not necessarily convex combinations of deterministic rules. 


\section{Leafless graphs}

In this section $G=(A, E)$ is a connected graph without leafs. The main result will be that every unanimous and strategy-proof PR is random dictatorial, to be defined below. We will derive this result for the case of two agents, and then use Theorem 5 in Chatterji et al (2014) to extend to more than two agents.

Our notational conventions about preferences as introduced in Section 2 will still be used. Additionally, for a path $\pi=\left[x_{1}, x_{\ell}\right]$ with sequence $x_{1}, \ldots, x_{\ell}$ we denote by $\pi^{-1}=\left[x_{\ell}, x_{1}\right]$ the path in reverse direction, i.e., with sequence $x_{\ell}, \ldots, x_{1}$, and use this in notations for preferences such as $P=\pi \cdots, P=\pi^{-1} \cdots$, etc., with obvious meaning.

A cycle in $G$ is a sequence of distinct alternatives $x_{1}, \ldots, x_{k} \in A$ for some $k \geq 2$ such that $\left\{\left\{x_{i}, x_{i+1}\right\},\left\{x_{k}, x_{1}\right\}: i=1, \ldots, k-1\right\} \subseteq E$.

The following lemma considers unanimous and strategy-proof PRs for the case of two agents.

Lemma 4.1. Let $n=2$ and let $\varphi: \mathcal{S}^{N} \rightarrow \Delta(A)$ be a unanimous and strategy-proof $P R$.

(i) Let $a, b \in A, a \neq b$, such that there is a cycle containing $a$ and $b$. Then there exists $\epsilon \in$ $[0,1]$ such that for all $P_{1}, P_{2} \in \mathcal{S}$ with $t\left(P_{1}\right)=a$ and $t\left(P_{2}\right)=b$ we have $\varphi_{a}\left(P_{1}, P_{2}\right)=\epsilon$ and $\varphi_{b}\left(P_{1}, P_{2}\right)=1-\epsilon$.

(ii) Let, additionally, $c \notin\{a, b\}$ such that there is a cycle containing $a$ and $c$, and a path from $b$ to $c$ not containing $a$. Then $\varphi_{a}\left(P_{1}, P_{2}\right)=\epsilon$ and $\varphi_{c}\left(P_{1}, P_{2}\right)=1-\epsilon$ for all $P_{1}, P_{2} \in \mathcal{S}$ with $t\left(P_{1}\right)=a$ and $t\left(P_{2}\right)=c$, with $\epsilon$ as in $(i)$.

Proof. (i) Since there is a cycle containing both $a$ and $b$, there exist two paths $\pi$ and $\hat{\pi}$ from $a$ to $b$ in $G$ such that $\pi \cap \hat{\pi}=\{a, b\}$. Hence, there are $P, Q \in \mathcal{S}$ such that $P=\pi \cdots$ and $Q=\hat{\pi}^{-1} \cdots$

Suppose that $\varphi_{c}(P, Q)>0$ for some $c \in A \backslash\{a, b\}$. Since $U(b, P) \cap U(a, Q)=\{a, b\}$, we have $c \notin U(b, P)$ or $c \notin U(a, Q)$. By unanimity, in the first case agent 1 can manipulate by changing to $Q$ and in the second case agent 2 can manipulate by changing to $P$. This contradicts strategy-proofness, and therefore we have $\varphi_{c}(P, Q)=0$ for all $c \in A \backslash\{a, b\}$. Thus, there exists $\epsilon \in[0,1]$ such that $\varphi_{a}(P, Q)=\epsilon$ and $\varphi_{b}(P, Q)=1-\epsilon$. Statement (i) now follows from tops-onliness of $\varphi$ (Lemma 2.6).

(ii) Let $P_{1}, P_{2} \in \mathcal{S}$ with $t\left(P_{1}\right)=a$ and $t\left(P_{2}\right)=c$. Assume that $\varphi_{a}\left(P_{1}, P_{2}\right)=\epsilon^{\prime}$. By a similar argument as in step (i), this implies $\varphi_{c}\left(P_{1}, P_{2}\right)=1-\epsilon^{\prime}$. Thus, it is sufficient to show that $\epsilon=\epsilon^{\prime}$. Suppose not. Assume without loss of generality that $\epsilon>\epsilon^{\prime}$. Let $\pi$ now be a path from $b$ to $c$ such that $a \notin \pi$, and consider associated preferences $P=\pi \cdots, P^{\prime}=\pi^{-1} \cdots \in \mathcal{S}$. By part (i), $\varphi_{U(c, P)}\left(P_{1}, P\right)=1-\epsilon<1-\epsilon^{\prime}=\varphi_{U(c, P)}\left(P_{1}, P^{\prime}\right)$. This violates strategy-proofness and, hence, $\epsilon=\epsilon^{\prime}$.

A PR $\varphi$ is random-dictatorial if there are $\alpha_{1}, \ldots, \alpha_{n} \in[0,1]$ with $\sum_{i \in N} \alpha_{i}=1$, such that for every $P_{N} \in \mathcal{S}^{N}$ and $a \in A$ we have $\varphi_{a}\left(P_{N}\right)=\sum_{i \in N: t\left(P_{i}\right)=a} \alpha_{i}$.

Clearly, a random dictatorial rule is unanimous and strategy-proof. Indeed, when $G$ is a tree, a random dictatorial rule is monotonic. To see this note that, if $\varphi$ is random dictatorial with weights $\alpha_{1}, \ldots, \alpha_{n}$, then for each leaf assignment $\mu \in \mathcal{M}$ define $\beta_{\mu}(a)=\sum_{i \in N: \mu(i)=a} \alpha_{i}$ for every $a \in L(G)$. Then the collection $\left(\beta_{\mu}\right)_{\mu \in \mathcal{M}}$ is monotonic and it is easy to verify that $\varphi=\varphi^{B}$. The following example provides an illustration of this. 
Example 4.2. Consider the following tree:

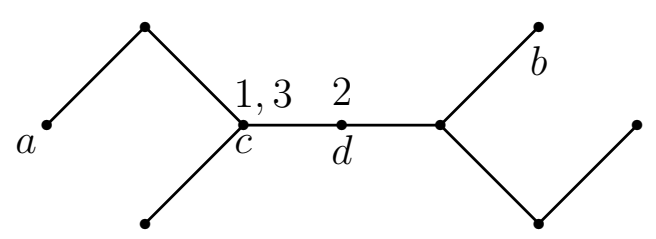

Let $N=\{1,2,3\}$ and let $\varphi$ be the random dictatorship with weights $\left(\alpha_{1}, \alpha_{2}, \alpha_{3}\right)=\left(\frac{1}{6}, \frac{1}{3}, \frac{1}{2}\right)$. The peaks of the agents in the profile $P_{N}$ are indicated in the picture. Hence $\varphi_{c}\left(P_{N}\right)=$ $\frac{1}{6}+\frac{1}{2}=\frac{2}{3}$ and $\varphi_{d}\left(P_{N}\right)=\frac{1}{3}$. With the collection $B$ defined as above, we obtain

$$
\begin{aligned}
\varphi_{c}^{B}\left(P_{N}\right) & =\beta_{\mu}([c, a])-\beta_{\hat{\mu}}((c, a]) \\
& =\frac{1}{6}+\frac{1}{2}-0=\frac{2}{3} \\
& =\varphi_{c}\left(P_{N}\right)
\end{aligned}
$$

where $\mu(1)=\mu(3)=a, \mu(2)=b$, and $\hat{\mu}(1)=\hat{\mu}(2)=\hat{\mu}(3)=b$. Similarly,

$$
\begin{aligned}
\varphi_{d}^{B}\left(P_{N}\right) & =\beta_{\mu^{\prime}}([d, a])-\beta_{\hat{\mu}^{\prime}}((d, a]) \\
& =1-\frac{1}{6}-\frac{1}{2}=\frac{1}{3} \\
& =\varphi_{d}\left(P_{N}\right)
\end{aligned}
$$

where $\mu^{\prime}(1)=\mu^{\prime}(2)=\mu^{\prime}(3)=a, \hat{\mu}^{\prime}(1)=\hat{\mu}^{\prime}(3)=a$, and $\hat{\mu}^{\prime}(2)=b$.

A graph $G$ is 2-connected if for all distinct $x, y \in A$ there is a cycle in $G$ containing $x$ and $y$. We can now state the following consequence of Lemma 4.1.

Lemma 4.3. Let $n=2$ and let $\varphi: \mathcal{S}^{N} \rightarrow \Delta(A)$ be a unanimous and strategy-proof PR. Assume that the graph $G$ is 2-connected. Then $\varphi$ is random dictatorial.

Proof. Let $a \in A$. By Lemma 4.1 there is an $\alpha \in[0,1]$ such that for all $x \in A$ and $P_{1}, P_{2} \in \mathcal{S}^{N}$ with $t\left(P_{1}\right)=a$ and $t\left(P_{2}\right)=x$ we have $\varphi_{a}\left(P_{1}, P_{2}\right)=\alpha$ and $\varphi_{x}\left(P_{1}, P_{2}\right)=1-\alpha$. Now let $b \in A, b \neq a$. Then similarly one proves that there is $\alpha^{\prime} \in[0,1]$ such that for all $x \in A$ and $Q_{1}, Q_{2} \in \mathcal{S}^{N}$ with $t\left(Q_{1}\right)=x$ and $t\left(Q_{2}\right)=b$ we have $\varphi_{b}\left(Q_{1}, Q_{2}\right)=\alpha^{\prime}$ and $\varphi_{x}\left(Q_{1}, Q_{2}\right)=1-\alpha^{\prime}$. Since the latter holds for $x=a$ in particular, we have $1-\alpha^{\prime}=\alpha$. This implies that for all $x, y \in A$ and $Z_{1}, Z_{2} \in \mathcal{S}^{N}$ with $t\left(Z_{1}\right)=x$ and $t\left(Z_{2}\right)=y$ we have $\varphi_{x}\left(Z_{1}, Z_{2}\right)=\alpha$ and $\varphi_{y}\left(Z_{1}, Z_{2}\right)=1-\alpha$. Hence, $\varphi$ is random dictatorial.

The following lemma shows that random dictatorship for $n=2$ still holds if the graph $G$ has no leaf.

Lemma 4.4. Let $n=2$, and let $G$ have no leaf. Let $\varphi: \mathcal{S}^{N} \rightarrow \Delta(A)$ be a unanimous and strategy-proof PR. Then $\varphi$ is random dictatorial.

Proof. If $G$ is 2-connected then the result follows from Lemma 4.3. Now assume that $G$ is not 2-connected. Since $G$ is connected we can decompose it into 2-connected subgraphs $\left(A_{1}, E_{1}\right), \ldots,\left(A_{\ell}, E_{\ell}\right)$, the set of remaining alternatives $B=A \backslash \cup_{i=1}^{\ell} A_{i}$ and the set of remaining edges $E^{\prime}=E \backslash \cup_{i=1}^{\ell} E_{i}{ }^{5}$

\footnotetext{
${ }^{5}$ This decomposition is close to the decomposition as a so-called block-tree. See, for instance, Bondy and Murty, 2008. The formal definition of a block-tree is slightly different, but the decomposition here is more convenient for our purposes.
} 
For any distinct $1 \leq p, q \leq \ell$ there are $a_{p} \in A_{p}$ and $a_{q} \in A_{q}$ such that all paths in $G$ from an alternative in $A_{p}$ to an alternative in $A_{q}$ leave $A_{p}$ via $a_{p}$ and enter $A_{q}$ via $a_{q}$. In this case, with some abuse of notation we use $\left[a_{p}, a_{q}\right]$ to denote the set of alternatives containing $a_{p}, a_{q}$, and all $x$ such that there is some path $\pi$ in $G$ with $x \in \pi$, starting at $a_{p}$ such that $\pi \cap A_{p}=\left\{a_{p}\right\}$, and $a_{q} \notin \pi$; or there is some path $\pi$ in $G$ with $x \in \pi$, starting at $a_{q}$ such that $\pi \cap A_{q}=\left\{a_{q}\right\}$, and $a_{p} \notin \pi$. Similarly, $\left[a_{p}, a_{q}\right)=\left[a_{p}, a_{q}\right] \backslash\left\{a_{q}\right\} ;\left[\leftarrow, a_{p}\right]$ denotes all alternatives on paths starting at $a_{p}$ which have only $a_{p}$ in common with $\left[a_{p}, a_{q}\right] ;\left[a_{q}, \rightarrow\right]$ denotes all alternatives on paths starting at $a_{q}$ which have only $a_{q}$ in common with $\left[a_{p}, a_{q}\right]$; $\left[\leftarrow, a_{q}\right]$ denotes all alternatives on paths starting at $a_{q}$ which have only $a_{q}$ in common with $A_{q}$; and so on and so forth. See the following diagram, which shows a possible part of the decomposition of $G$, and visualizes parts of rest of the proof.

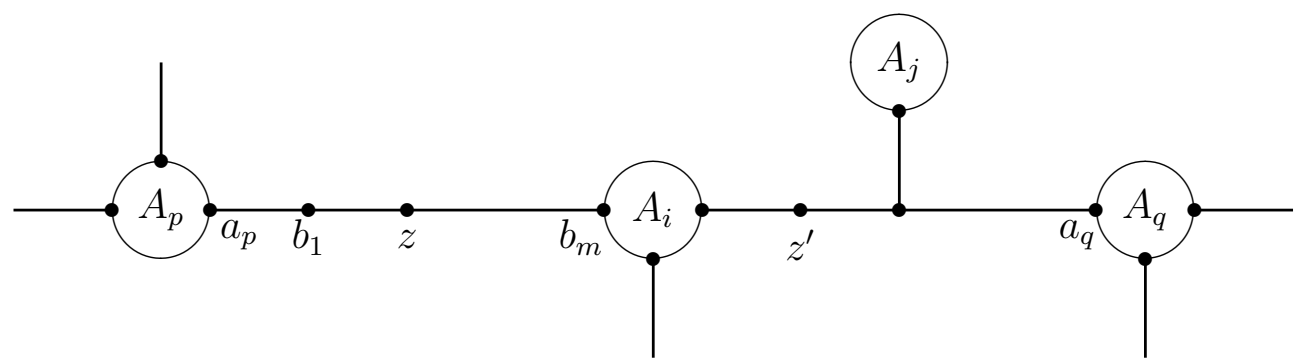

By (the proof of) Lemma 4.3 there are $\alpha_{1}, \ldots, \alpha_{\ell} \in[0,1]$ such that, for all $i=1, \ldots, \ell$, $\varphi_{t\left(P_{1}\right)}\left(P_{1}, P_{2}\right)=\alpha_{i}$ and $\varphi_{t\left(P_{2}\right)}\left(P_{1}, P_{2}\right)=1-\alpha_{i}$ for all $\left(P_{1}, P_{2}\right) \in \mathcal{S}^{N}$ with $t\left(P_{1}\right), t\left(P_{2}\right) \in A_{i}$. (In words, $\varphi$ induces a random dictatorship on every $A_{i}$.) The proof proceeds in three steps.

(a) With notations as above, we first consider a profile $\left(P_{1}, P_{2}\right)$ such that $t\left(P_{1}\right) \in A_{p} \backslash\left\{a_{p}\right\}$ and $t\left(P_{2}\right) \in A_{q} \backslash\left\{a_{q}\right\}$ for some $1 \leq p<q \leq \ell$. Since $\varphi_{a_{q}}\left(P_{1}^{\prime}, P_{2}\right)=\alpha_{q}$ for $P_{1}^{\prime} \in \mathcal{S}$ with $t\left(P_{1}^{\prime}\right)=a_{q}$, strategy-proofness (considering agent 1 ) implies that

$$
\varphi_{\left[\leftarrow, a_{q}\right]}\left(P_{1}, P_{2}\right) \geq \alpha_{q}
$$

Similarly,

$$
\varphi_{\left[a_{p}, \rightarrow\right]}\left(P_{1}, P_{2}\right) \geq 1-\alpha_{p}
$$

Now consider $\tilde{P}_{1} \in \mathcal{S}$ with $t\left(\tilde{P}_{1}\right) \in A_{q} \backslash\left\{t\left(P_{2}\right)\right\}$ and such that $x \tilde{P}_{1} t\left(P_{2}\right)$ for all $x \in\left[\leftarrow, a_{q}\right]$. Let $y \in\left[\leftarrow, a_{q}\right]$ such that $x \tilde{P}_{1} y$ for all $x \in\left[\leftarrow, a_{q}\right]$. Since $\varphi_{t\left(\tilde{P}_{1}\right)}\left(\tilde{P}_{1}, P_{2}\right)=\alpha_{q}$, strategy-proofness (considering agent 1 ) requires that $\varphi_{U\left(y, \tilde{P}_{1}\right)}\left(P_{1}, P_{2}\right) \leq \alpha_{q}$, hence:

$$
\varphi\left[\leftarrow, a_{q}\right]\left(P_{1}, P_{2}\right) \leq \alpha_{q} .
$$

Similarly,

$$
\varphi_{\left[a_{p}, \rightarrow\right]}\left(P_{1}, P_{2}\right) \leq 1-\alpha_{p}
$$

Combining (10) and (12) we obtain $\varphi_{\left[\leftarrow, a_{q}\right]}\left(P_{1}, P_{2}\right)=\alpha_{q}$, and combining (11) and (13) we obtain $\varphi_{\left[a_{p}, \rightarrow\right]}\left(P_{1}, P_{2}\right)=1-\alpha_{p}$. By adding up these two equalities it follows that $\varphi_{\left[a_{p}, a_{q}\right]}\left(P_{1}, P_{2}\right)=\alpha_{q}-\alpha_{p}$. Similarly one proves $\varphi_{\left[a_{p}, a_{q}\right]}\left(P_{1}, P_{2}\right)=\alpha_{p}-\alpha_{q}$. Hence, $\alpha_{p}=\alpha_{q}$ and $\varphi_{\left[a_{p}, a_{q}\right]}\left(P_{1}, P_{2}\right)=0$. Now writing $\alpha$ for $\alpha_{1}, \ldots, \alpha_{\ell}$, we obtain by (10) and (11) that $\varphi_{\left[\leftarrow, a_{p}\right)}\left(P_{1}, P_{2}\right)=\alpha$ and $\varphi_{\left(a_{q}, \rightarrow\right]}\left(P_{1}, P_{2}\right)=1-\alpha$.

We next show that $\varphi_{t\left(P_{1}\right)}\left(P_{1}, P_{2}\right)=\alpha$. Consider two paths $\pi$ and $\sigma$ in $G$ from $t\left(P_{1}\right)$ to $a_{p}$ with all alternatives in $A_{p}$ and which only have $t\left(P_{1}\right)$ and $a_{p}$ in common, and let $P_{1}^{\prime} \in \mathcal{S}$ with $P_{1}^{\prime}=\pi \cdots$. By strategy-proofness ${ }^{6}$ (considering agent 1 ), it is sufficient to prove that

$$
\varphi_{t\left(P_{1}^{\prime}\right)}\left(P_{1}^{\prime}, P_{2}\right)=\alpha \text {. }
$$

${ }^{6}$ Or by tops-onliness, Lemma 2.6 . 
Since $\varphi_{\left[\leftarrow, a_{p}\right)}\left(P_{1}, P_{2}\right)=\alpha$ and $\varphi_{a_{q}}\left(\hat{P}_{1}, P_{2}\right)=\alpha$ for $\hat{P}_{1} \in \mathcal{S}$ with $t\left(\hat{P}_{1}\right)=a_{q}$, by strategyproofness we have $\varphi_{\pi}\left(P_{1}^{\prime}, P_{2}\right)=\alpha$. Suppose that there is a $v \in \pi, v \neq t\left(P_{1}^{\prime}\right), v \neq a_{p}$, such that

$$
\varphi_{v}\left(P_{1}^{\prime}, P_{2}\right)>0
$$

Consider $P_{2}^{\prime} \in \mathcal{S}$ with $t\left(P_{2}^{\prime}\right) \in A_{q}$ and with $P_{2}^{\prime}=\cdots x \cdots\left(\pi^{-1} \backslash\left\{a_{p}, t\left(P_{1}\right)\right\}\right)\left(\sigma \backslash\left\{a_{p}\right\}\right) \cdots$ for all $x \in\left[a_{p}, \rightarrow\right]$. (Hence, $P_{2}^{\prime}$ orders all alternatives 'to the right' of $a_{p}$ before $a_{p}$, then the alternatives on path $\pi$ in reverse order, next the alternatives on path $\sigma$ up to but not including $a_{p}$, and finally all remaining alternatives.) By (15) and strategy-proofness,

$$
\varphi_{\left[v, a_{p}\right)}\left(P_{1}^{\prime}, P_{2}^{\prime}\right)>0
$$

where $\left[v, a_{p}\right)$ denotes the part of path $\pi$ from $v$ up to but excluding the end point $a_{p}$. Next consider $P_{1}^{\prime \prime} \in \mathcal{S}$ with $P_{1}^{\prime \prime}=\sigma \cdots$. Then by strategy-proofness $\varphi_{\sigma}\left(P_{1}^{\prime \prime}, P_{2}^{\prime}\right)=\alpha$ (otherwise agent 1 manipulates), which again by strategy-proofness implies $\varphi_{t\left(P_{1}^{\prime}\right)}\left(P_{1}^{\prime \prime}, P_{2}^{\prime}\right)=\alpha$ (otherwise agent 2 manipulates). In turn, by strategy-proofness this implies $\varphi_{t\left(P_{1}^{\prime}\right)}\left(P_{1}^{\prime}, P_{2}^{\prime}\right)=\alpha$ (otherwise agent 1 manipulates), which contradicts (16). Consequently, (15) does not hold, which implies (14).

Similarly, one proves that $\varphi_{t\left(P_{2}\right)}\left(P_{1}, P_{2}\right)=1-\alpha$.

(b) Second, all paths in $G$ from $a_{p}$ to $a_{q}$ have a common initial part which is either (i) only $a_{p}$ or (ii) $\left[a_{p}, b_{1}, \ldots, b_{m}\right]$ for some $m \geq 1$ with $b_{1}, \ldots, b_{m-1} \in B$. Let now $\left(P_{1}, P_{2}\right)$ be a profile with $t\left(P_{2}\right) \in A_{q} \backslash\left\{a_{q}\right\}$ and $t\left(P_{1}\right)=z$, where $z=a_{p}$ in case (i), or $z \in\left[a_{p}, b_{1}, \ldots, b_{m}\right)$ in case (ii). By strategy-proofness (considering agent 1) and part (a), we have $\varphi_{\left[\leftarrow, a_{q}\right]}\left(P_{1}, P_{2}\right)=\alpha$. By strategy-proofness (considering agent 2) and unanimity, $\varphi_{[z, \rightarrow]}\left(P_{1}, P_{2}\right)=1$. Therefore, $\varphi_{\left[z, a_{q}\right]}\left(P_{1}, P_{2}\right)=\alpha$ and $\varphi_{\left(a_{q}, \rightarrow\right]}\left(P_{1}, P_{2}\right)=1-\alpha$.

Consider $P_{1}^{\prime} \in \mathcal{S}$ with $P_{1}^{\prime}=\left[z, a_{p}\right] \cdots x \cdots y \cdots$ for all $x \in\left[\leftarrow, a_{p}\right)$ and all $y \in(z, \rightarrow]$. Then as before $\varphi_{\left[z, a_{q}\right]}\left(P_{1}^{\prime}, P_{2}\right)=\alpha$, which together with part (a) and strategy-proofness (considering agent 1 ) implies $\varphi_{z}\left(P_{1}^{\prime}, P_{2}\right)=\alpha$. In turn, by strategy-proofness (considering agent 1) this implies $\varphi_{t\left(P_{1}\right)}\left(P_{1}, P_{2}\right)=\varphi_{z}\left(P_{1}, P_{2}\right)=\alpha$.

Suppose $\varphi_{b}\left(P_{1}, P_{2}\right)>0$ for some $b \in\left(a_{q}, \rightarrow\right]$ with $b \neq t\left(P_{2}\right)$. Then consider $\tilde{P}_{1} \in \mathcal{S}$ with $t\left(\tilde{P}_{1}\right) \in A_{p} \backslash\left\{a_{p}\right\}$ and $b \tilde{P}_{1} t\left(P_{2}\right)$. Then agent 1 with preference $\tilde{P}_{1}$ manipulates via $P_{1}$, a contradiction. Hence, $\varphi_{t\left(P_{2}\right)}\left(P_{1}, P_{2}\right)=1-\alpha$.

Similarly one proves $\varphi_{t\left(P_{1}\right)}\left(P_{1}, P_{2}\right)=\alpha$ and $\varphi_{t\left(P_{2}\right)}\left(P_{1}, P_{2}\right)=1-\alpha$ if $t\left(P_{1}\right) \in A_{p} \backslash\left\{a_{p}\right\}$ and $t\left(P_{2}\right)=z^{\prime}$, where $z^{\prime}$ is an alternative on the common initial part of all paths from $a_{q}$ to $a_{p}$, analogously as above.

(c) Finally, let $\left(P_{1}, P_{2}\right)$ be a profile with $t\left(P_{1}\right)=z$ and $t\left(P_{2}\right)=z^{\prime}$ with $z$ and $z^{\prime}$ as in part (b). By unanimity and strategy-proofness, $\varphi_{\left[z, z^{\prime}\right]}\left(P_{1}, P_{2}\right)=1$. In order to prove that $\varphi_{z}\left(P_{1}, P_{2}\right)=\alpha$ and $\varphi_{z^{\prime}}\left(P_{1}, P_{2}\right)=1-\alpha$ it is therefore sufficient to prove that $\varphi_{z}\left(P_{1}, P_{2}\right) \geq \alpha$. Consider $P_{1}^{\prime} \in \mathcal{S}$ as in (b), i.e., $P_{1}^{\prime} \in \mathcal{S}$ with $P_{1}^{\prime}=\left[z, a_{p}\right] \cdots x \cdots y \cdots$ for all $x \in\left[\leftarrow, a_{p}\right)$ and all $y \in(z, \rightarrow]$. By strategy-proofness (considering agent 1 ) and part (b), $\varphi_{z}\left(P_{1}^{\prime}, P_{2}\right) \geq \alpha$. By strategy-proofness this implies $\varphi_{z}\left(P_{1}, P_{2}\right) \geq \alpha$, as was to be proved.

Theorem 5 in Chatterji et al (2014) states that if, for $n=2$, every unanimous and strategy-proof $\mathrm{PR}$ on a domain satisfying 'Condition $\alpha$ ' is random dictatorial, then the same is true for $n>2$. This Condition $\alpha$ requires that there are distinct alternatives $a, b, c \in A$ and preferences $P_{1}, P_{2}$, and $P_{3}$, such that (i) $P_{1}=a \cdots b \cdots c \cdots, P_{2}=b \cdots c \cdots a \cdots$, and $P_{3}=c \cdots a \cdots b \cdots$, and (ii) for every $x \in A \backslash\{a, b, c\}, c P_{1} x$ or $a P_{2} x$ or $b P_{3} x$. It is not hard 
to verify that Condition $\alpha$ holds if $G$ does not have a leaf. ${ }^{7}$ Hence, by Lemma 4.4 , we have the following result.

Lemma 4.5. Let $\varphi: \mathcal{S}^{N} \rightarrow \Delta(A)$ be a unanimous and strategy-proof $P R$, and let the graph $G$ have no leaf. Then $\varphi$ is random dictatorial.

If $G$ has a leaf, then a unanimous and strategy-proof PR is not necessarily random dictatorial, as the following lemma shows.

Lemma 4.6. Let $G$ have a leaf. Then there exists a unanimous and strategy-proof PR which is not random dictatorial.

Proof. Let $x \in A$ be a leaf and let $y \in A$ with $\{x, y\} \in E$. Let $\alpha_{1}, \ldots, \alpha_{n} \in[0,1]$ with $\sum_{i \in N} \alpha_{i}=1$. For every $P_{N} \in \mathcal{S}^{N}$ such that $t\left(P_{i}\right) \neq x$ for some $i \in N$ and every $a \in A \backslash\{x, y\}$ define $\varphi_{a}\left(P_{N}\right)=\sum_{i \in N: t\left(P_{i}\right)=a} \alpha_{i}$, and define $\varphi_{y}\left(P_{N}\right)=\sum_{i \in N: t\left(P_{i}\right) \in\{x, y\}} \alpha_{i}$. For every $P_{N} \in \mathcal{S}^{N}$ such that $t\left(P_{i}\right)=x$ for every $i \in N$ define $\varphi_{x}\left(P_{N}\right)=1$. Then it is straightforward to verify that $\varphi$ is unanimous and strategy-proof. However, $\varphi$ is not random dictatorial.

In fact, in the next section, for general connected graphs, all unanimous and strategyproof PRs are characterized. For now, combining Lemmas 4.5 and 4.6, we obtain the main result of this section.

Theorem 4.7. Let $G$ be a connected graph. Then every unanimous and strategy-proof PR on $\mathcal{S}^{N}$ is random dictatorial if and only if $G$ has no leaf.

\section{General connected graphs}

Throughout the section, $G=(A, E)$ is an arbitrary connected graph. Let $\bar{G}=(\bar{A}, \bar{E})$ denote the maximal subgraph of $G$ (i.e., $\bar{E} \subseteq E$ and $\bar{A}=\{a \in A:\{a, b\} \in \bar{E}$ for some $b \in A\}$ ) that has no leaf. Observe that $\bar{G}$ is unique, and $\bar{G}=\emptyset$ (i.e., $\bar{A}=\bar{E}=\emptyset$ ) if and only if $G$ is a tree.

For each leaf $l \in L(G)$, the set of alternatives $A(l) \subseteq A$ and the set of edges $E(l) \subseteq E$ are defined by

$$
A(l)=\{l\} \cup\{a \in A: \text { there is a path }[a, l] \text { such that }|[a, l] \cap \bar{A}| \leq 1\},
$$

and

$$
E(l)=\{\{a, b\} \in E: a, b \in A(l)\} .
$$

The subgraph $(A(l), E(l))$ is called the branch of $l$. Observe that $A(l)$ has a unique alternative in common with $\bar{A}$, which we denote by $a(l)$. We also denote $\bar{A}^{0}=\bar{A} \backslash\{a(l): l \in L(G)\}$. Thus, $\bar{A}^{0}$ together with the sets $A(l)$ for $l \in L(G)$ form a partition of $A$. (For an illustration of these concepts see Example 5.7 below.)

In this section we characterize all unanimous and strategy-proof PRs. We start with the following auxiliary lemma.

\footnotetext{
${ }^{7}$ If $G$ does not have a leaf, it has a cycle. Take three adjacent alternatives $a, b, c$ on this cycle and take a spanning tree $T=\left(A, E_{T}\right)$ with $\{a, b\},\{b, c\} \in E_{T}$. Take preferences $P_{1}=a b c \cdots$ and $P_{2}=b c a \cdots$. Take another spanning tree including a path from $c$ to $a$ that does not contain $b$, and take a preference $P_{3}=c \cdots a \cdots b \cdots$. Then $a, b, c$ and $P_{1}, P_{2}, P_{3} \in \mathcal{S}^{N}$ satisfy Condition $\alpha$.
} 
Lemma 5.1. Let $i \in N, P_{i} \in \mathcal{S}, P_{-i} \in \mathcal{S}^{N \backslash\{i\}}$, and $x, y \in A$ such that $\{x, y\} \in E$ and $t\left(P_{i}\right)=x$. Let $P_{i}^{\prime}=y x \cdots \in \mathcal{S}$ such that $a P_{i}^{\prime} b \Leftrightarrow a P_{i} b$ for all $a, b \in A \backslash\{x, y\}$. Let $\varphi$ be $a$ unanimous and strategy-proof $P R$. Then $\varphi_{a}\left(P_{i}, P_{-i}\right)=\varphi_{a}\left(P_{i}^{\prime}, P_{-i}\right)$ for all $a \notin U\left(y, P_{i}\right)$.

Proof. Write $P_{i}=x b_{1} \cdots b_{k} y a_{1} \cdots a_{\ell}$, then $P_{i}^{\prime}=y x b_{1} \cdots b_{k} a_{1} \cdots a_{\ell}$. By strategy-proofness, $\varphi_{U\left(a_{\ell-1}, P_{i}\right)}\left(P_{i}, P_{-i}\right) \geq \varphi_{U\left(a_{\ell-1}, P_{i}\right)}\left(P_{i}^{\prime}, P_{-i}\right)$ and $\varphi_{U\left(a_{\ell-1}, P_{i}^{\prime}\right)}\left(P_{i}^{\prime}, P_{-i}\right) \geq \varphi_{U\left(a_{\ell-1}, P_{i}^{\prime}\right)}\left(P_{i}, P_{-i}\right)$, hence $\varphi_{a_{\ell}}\left(P_{i}, P_{-i}\right)=\varphi_{a_{\ell}}\left(P_{i}^{\prime}, P_{-i}\right)$. Repeating this argument we obtain $\varphi_{a_{j}}\left(P_{i}, P_{-i}\right)=\varphi_{a_{j}}\left(P_{i}^{\prime}, P_{-i}\right)$ for all $j=1, \ldots, \ell$.

The next lemma shows that a unanimous and strategy-proof $\mathrm{PR} \varphi$ is a random dictatorship when restricted to profiles with all peaks in $\bar{A}$.

Lemma 5.2. Let $\varphi$ be a unanimous and strategy-proof $P R$. Then there exist $\alpha_{1}, \ldots, \alpha_{n} \geq 0$ with $\sum_{i=1}^{n} \alpha_{i}=1$ such that $\varphi_{a}\left(P_{N}\right)=\sum_{i \in N: t\left(P_{i}\right)=a} \alpha_{i}$ for all $a \in \bar{A}$ and all $P_{N} \in \mathcal{S}^{N}$ with $t\left(P_{i}\right) \in \bar{A}$ for all $i \in N$.

Proof. Let $P_{N} \in \mathcal{S}^{N}$ with $t\left(P_{i}\right) \in \bar{A}$ for all $i \in N$. Suppose that $\varphi_{A(l) \backslash\{a(l)\}}\left(P_{N}\right)>0$ for some $l \in L(G)$. Consider $i \in N$ and let $T$ be a spanning tree of $G$ such that $P_{i}$ is single-peaked with respect to $T$. Let $x=t\left(P_{i}\right)$ and suppose that $x \neq a(l)$. Take $y \in \bar{A}$ such that $\{x, y\}$ is an edge of $T$ and $y$ is on the path from $x$ to $a(l)$ in $T$. Let $P_{i}^{\prime}$ be derived from $P_{i}$ as in Lemma 5.1, i.e., $P_{i}^{\prime}=y x \cdots a(l) \cdots, P_{i}=x \cdots y \cdots a(l) \cdots$, and $P_{i}$ and $P_{i}^{\prime}$ order all alternatives different from $x$ and $y$ equally. Then Lemma 5.1 implies that $\varphi_{a}\left(P_{i}^{\prime}, P_{-i}\right)=\varphi_{a}\left(P_{i}, P_{-i}\right)$ in particular for all $a \in A(l)$. By repeatedly applying this argument for player $i$ and for all other players we arrive at a profile $\tilde{P}_{N}$ with $t\left(\tilde{P}_{j}\right)=a(l)$ for every $j \in N$ and still $\varphi_{A(l) \backslash\{a(l)\}}\left(\tilde{P}_{N}\right)>0$, which contradicts unanimity of $\varphi$. Hence, $\varphi_{\bar{A}}\left(P_{N}\right)=1$.

Next, for all $a(l) \in \bar{A}$, let $P^{l}$ be a single-peaked preference on $A(l)$ with graph (tree) $(A(l), E(l))$ and peak $a(l)$. For any single-peaked preference $\bar{P}$ on $(\bar{A}, \bar{E})$, construct the single-peaked preference $\bar{P}^{e}$ on $G$ by substituting, in $\bar{P}$, each $a(l)$ by $P^{l}$. Now define the PR $\bar{\varphi}$ on $(\bar{A}, \bar{E})$ by

$$
\bar{\varphi}\left(\bar{P}_{N}\right)=\varphi\left(\bar{P}_{N}^{e}\right)
$$

for each $\bar{P}_{N}$ on $\bar{A}$ which is single-peaked with respect to $(\bar{A}, \bar{E})$. By the first part of the proof, $\bar{\varphi}$ is well-defined, i.e., $\bar{\varphi}_{\bar{A}}\left(\bar{P}_{N}\right)=1$ for all $\bar{P}_{N}$. Also, it inherits unanimity and strategyproofness from $\varphi$. By Theorem 4.7 it follows that there are $\alpha_{1}, \ldots, \alpha_{n} \geq 0$ with $\sum_{i=1}^{n} \alpha_{i}=1$ such that $\bar{\varphi}_{a}\left(\bar{P}_{N}\right)=\sum_{i \in N: t\left(\bar{P}_{i}\right)=a} \alpha_{i}$ for all $a \in \bar{A}$ and each $\bar{P}_{N}$ consisting of preferences that are single-peaked with respect to $(\bar{A}, \bar{E})$. By $(17)$, the proof of the lemma is complete by observing that, due to tops-onliness (Lemma 2.6), $\bar{\varphi}$ does not depend on the particular extension $\bar{P}^{e}$ of $\bar{P}$.

The next lemma extends the previous one by also including the branches of $G$.

Lemma 5.3. Let $\varphi$ be a unanimous and strategy-proof $P R$. Then there exist $\alpha_{1}, \ldots, \alpha_{n} \geq 0$ with $\sum_{i=1}^{n} \alpha_{i}=1$ such that for all $a \in \bar{A}^{0}$ and all $l \in L(G)$

$$
\varphi_{a}\left(P_{N}\right)=\sum_{i \in N: t\left(P_{i}\right)=a} \alpha_{i}
$$

and

$$
\varphi_{A(l)}\left(P_{N}\right)=\sum_{i \in N: t\left(P_{i}\right) \in A(l)} \alpha_{i}
$$

for every $P_{N} \in \mathcal{S}_{N}$. 
Proof. Let $P_{N} \in \mathcal{S}^{N}$ and suppose that $i \in N$ and $t\left(P_{i}\right)=x \in A(l) \backslash\{a(l)$ for some $l \in L(G)$. Consider $P_{i}^{\prime}$ with $t\left(P_{i}^{\prime}\right)=y$ such that $\{x, y\} \in E, y$ on the path from $x$ to $a(l)$, as in Lemma 5.1. By this lemma, we obtain that $\varphi_{a}\left(P_{i}^{\prime}, P_{-i}\right)=\varphi_{a}\left(P_{N}\right)$ for all $a \notin A(l) \backslash\{a(l)\}$. The proof is complete by repeating this argument for agent $i$ and all other agents, and next applying Lemma 5.2.

We now fix a spanning tree $T=\left(A, E_{T}\right)$ of the graph $G=(A, E)$. Clearly, $L(G) \subseteq L(T)$, i.e., each leaf of $G$ is still a leaf of $T$. For $l \in L(T) \backslash L(G)$ define $A(l)=\{l\}$. The set of preferences on $A$ that are single-peaked with respect to $T$ is denoted by $\mathcal{S}_{T}$. Let $\mathcal{M}$ denote the set of leaf assignments with respect to $T$ (cf. Section 4). The next lemma connects a unanimous and strategy-proof $\operatorname{PR} \varphi$ to a monotonic $\operatorname{PR} \varphi^{B}$, as defined in Section 4 .

Lemma 5.4. Let $\varphi$ be a unanimous and strategy-proof $P R$ on $\mathcal{S}^{N}$, and let $\tilde{\varphi}$ denote the restriction of $\varphi$ to $\mathcal{S}_{T}^{N}$. Then there are $\alpha_{1}, \ldots, \alpha_{n} \geq 0$ with $\sum_{i=1}^{n} \alpha_{i}=1$ and a monotonic collection of probability distributions $B=\left(\beta_{\mu}\right)_{\mu \in \mathcal{M}}$ with

$$
\beta_{\mu}(A(l))=\sum_{i \in N: \mu(i) \in A(l)} \alpha_{i} \text { for every } l \in L(T) \text { and } \mu \in \mathcal{M}
$$

such that $\tilde{\varphi}=\varphi^{B}$.

Proof. Let the numbers $\alpha_{1}, \ldots, \alpha_{n}$ be as in Lemma 5.2. Clearly, $\tilde{\varphi}$ defined on $\mathcal{S}_{T}^{N}$ is unanimous and strategy-proof, and thus by Lemma 3.12 there is a monotonic collection of probability distributions $B=\left(\beta_{\mu}\right)_{\mu \in \mathcal{M}}$ such that $\tilde{\varphi}=\varphi^{B}$. We are left to show (18). Let $\mu \in \mathcal{M}$ and take $P_{N} \in \mathcal{S}_{T}^{N}$ such that $t\left(P_{i}\right)=\mu(i)$ for every $i \in N$.

(i) First consider $l \in L(G)$, and consider $\hat{\mu} \in \mathcal{M}$ such that $\hat{\mu}(i)=\mu(i)$ for all $i \in N$ with $\mu(i) \neq l$, and with $\hat{\mu}(i) \neq l$ for all $i \in N$ with $\mu(i)=l$. Then $\mu, \hat{\mu} \in \mathcal{M}\left(l, P_{N}\right)$ and by (4) we obtain

$$
\varphi_{l}^{B}\left(P_{N}\right)=\beta_{\mu}(\{l\})-\beta_{\hat{\mu}}(\emptyset)=\beta_{\mu}(\{l\}) .
$$

Again by (4), for $a \in A(l) \backslash L(G)$,

$$
\varphi_{a}^{B}\left(P_{N}\right)=\beta_{\mu}([a, l])-\beta_{\mu}((a, l])=\beta_{\mu}(\{a\}),
$$

where $[a, l]$ and $(a, l]$ are paths in $T$. By (19) and (20) we obtain for each $l \in L(G)$

$$
\beta_{\mu}(A(l))=\sum_{l^{\prime} \in A(l) \cap L(G)} \beta_{\mu}\left(\left\{l^{\prime}\right\}\right)+\sum_{a \in A(l) \backslash L(G)} \beta_{\mu}(\{a\})=\varphi_{A(l)}^{B}\left(P_{N}\right),
$$

hence by the definition of $\tilde{\varphi}=\varphi^{B}$ and Lemma 5.3

$$
\beta_{\mu}(A(l))=\varphi_{A(l)}\left(P_{N}\right)=\sum_{i \in N: \mu(i) \in A(l)} \alpha_{i} .
$$

(ii) Second consider $l \in L(T) \backslash L(G)$. Similarly as in (i) we obtain $\beta_{\mu}(A(l))=\beta_{\mu}(\{l\})=$ $\varphi_{l}^{B}\left(P_{N}\right)$, which by Lemma 5.3 implies

$$
\beta_{\mu}(A(l))=\sum_{i \in N: \mu(i)=l} \alpha_{i}
$$

Now (18) follows from (22) and (23). 
We can now state and prove the main and most general result of this paper.

Theorem 5.5. Let $G=(A, E)$ be a connected graph and let $T$ be a spanning tree of $G$. $A$ $\operatorname{PR} \varphi$ on $\mathcal{S}^{N}$ is unanimous and strategy-proof if and only if there are $\alpha_{1}, \ldots, \alpha_{n} \geq 0$ with $\sum_{i=1}^{n} \alpha_{i}=1$ and a monotonic collection of probability distributions $B=\left(\beta_{\mu}\right)_{\mu \in \mathcal{M}}$ with

$$
\beta_{\mu}(A(l))=\sum_{i \in N: \mu(i) \in A(l)} \alpha_{i} \text { for every } l \in L(T)
$$

such that $\varphi\left(P_{N}\right)=\varphi^{B}\left(\tilde{P}_{N}\right)$ for all tops-equivalent $P_{N} \in \mathcal{S}^{N}$ and $\tilde{P}_{N} \in \mathcal{S}_{T}^{N}$.

Proof. The only-if direction follows from Lemmas 5.4 and 2.6. For the if-direction, with $\left(\alpha_{i}\right)_{i \in N}$ and $B$ as in the statement of the theorem, define the $\operatorname{PR} \varphi$ on $\mathcal{S}^{N}$ by $\varphi\left(P_{N}\right)=\varphi^{B}\left(\tilde{P}_{N}\right)$ for every $P_{N} \in \mathcal{S}^{N}$, where $\tilde{P}_{N} \in \mathcal{S}_{T}^{N}$ is arbitrary but tops-equivalent to $P_{N}$. Clearly, since $\varphi^{B}$ is tops-only by Lemma 2.6 and Theorem 3.13, $\varphi$ is well-defined. It is straightforward to check that $\varphi$ is unanimous and strategy-proof.

Theorem 5.5 indeed generalizes Theorems 3.13 and 4.7, as we show in the following remark.

Remark 5.6. (i) If $G$ is a tree, then $T=G$ and $A(l)=A$ for all $l \in L(G)$. In this case one can take $\alpha_{1}, \ldots, \alpha_{n}$ arbitrary and (24) is trivially satisfied. Thus, Theorem 5.5 reduces to Theorem 3.13. (ii) If $G$ has no leaf, then $A(l)=\{l\}$ for every $l \in L(T)$. Now (24) and the definition of $\varphi^{B}$ imply that $\varphi$ is a random dictatorship with weights $\alpha_{1}, \ldots, \alpha_{n}$. Thus, Theorem 5.5 reduces to Theorem 4.7 .

We conclude the section with a few examples illustrating Theorem 5.5.

Example 5.7. Consider the following graph:

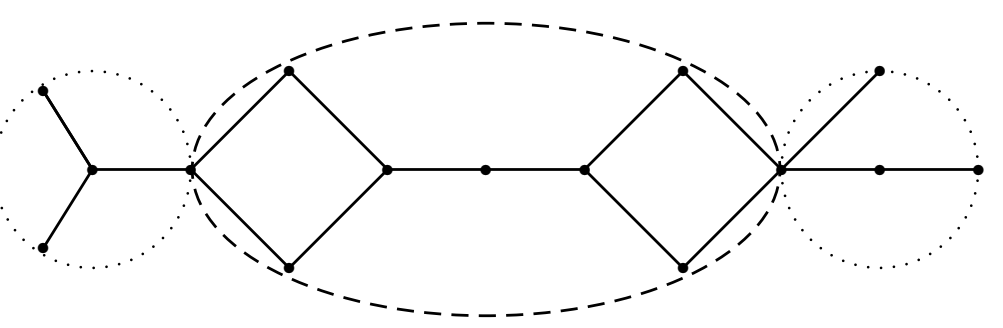

This graph has two branches (within the dotted circles), and the maximal leafless subgraph is the middle part (within the dashed oval). We take an arbitrary spanning tree:

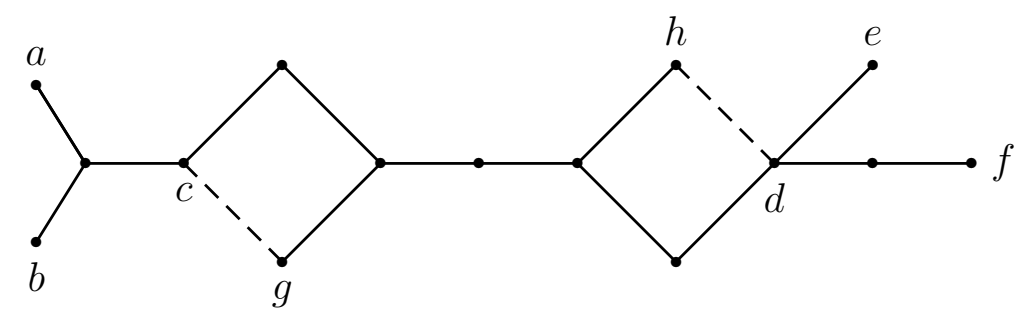

Now every unanimous and strategy-proof probabilistic rule is of the form $\varphi^{B}$, where $B=$ $\left(\beta_{\mu}\right)_{\mu \in \mathcal{M}}$ is a monotonic collection of probability distributions for this spanning tree satisfying, for every $\mu \in \mathcal{M}$,

$$
\beta_{\mu}(x)=\sum_{i \in N: \mu(i)=x} \alpha_{i} \text { for every } x \in\{g, h\}
$$


and

$$
\left.\beta_{\mu}(\{x, y, z\})=\sum_{i \in N: \mu(i) \in\{x, y, z\}} \alpha_{i} \text { for }\{x, y, z\}=\{a, b, c\} \text { and }\{x, y, z\}=\{d, e, f\}\right\}
$$

for weights $\alpha_{1}, \ldots, \alpha_{n}$.

Example 5.8. Consider the following graph and (on the right) a spanning tree:
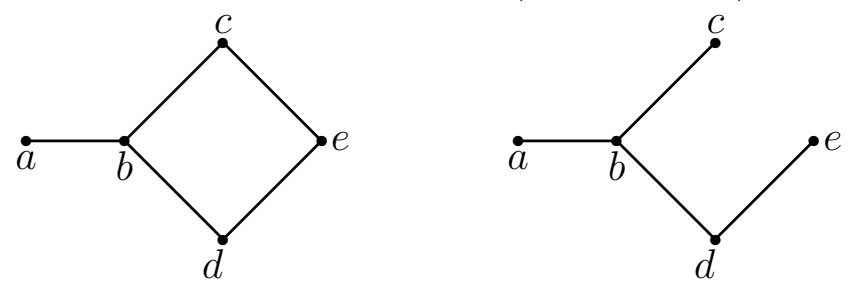

Let $N=\{1,2,3\}, \alpha_{1}=\alpha_{2}=\alpha_{3}=\frac{1}{3}$, and let each $\beta_{\mu}$ assign equal probabilities to $a$ and $b$ if the number of agents assigned to $a$ is below 3. Then, for instance, if $P_{N} \in \mathcal{S}^{N}$ satisfies $t\left(P_{1}\right)=a, t\left(P_{2}\right)=c$, and $t\left(P_{3}\right)=d$, then $\varphi^{B}$ assigns $\left(\frac{1}{6}, \frac{1}{6}, \frac{1}{3}, \frac{1}{3}, 0\right)$ to $(a, b, c, d, e)$.

\section{Random and deterministic rules}

Let $G=(A, E)$ be a tree. The collection of monotonic PRs characterized in Theorem 3.13 contains deterministic rules, i.e., rules that assign probability one to some alternative. It is not difficult to verify that these deterministic rules correspond to monotonic collections $B=\left(\beta_{\mu}\right)_{\mu \in \mathcal{M}}$ which are deterministic, that is, for every $\mu \in \mathcal{M}, \beta_{\mu}(x)=1$ for some $x \in A$.

The following example shows that, in contrast to the case where the graph is a line graph (Peters et al., 2014) not every monotonic PR can be written as a convex combination of deterministic monotonic rules.

Example 6.1. Let $N=\{1,2,3\}$ and $A=\{a, b, c, d\}$, and let $G=(A, E)$ be the tree below. We consider the anonymous monotonic rule with monotonic collection of leaf assignments as in the following table, in which $\beta_{(j, k, l)}$ denotes the probabilities assigned by the leaf assignment where $j$ agents are assigned to $a, k$ agents to $b$, and $l$ agents to $c$.

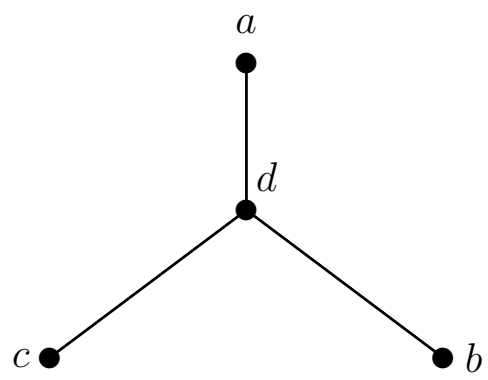

\begin{tabular}{|l|c|c|c|c|}
\hline & $a$ & $b$ & $c$ & $d$ \\
\hline$\beta_{(1,1,1)}$ & .5 & .3 & .2 & 0 \\
\hline$\beta_{(2,1,0)}$ & .7 & .3 & 0 & 0 \\
\hline$\beta_{(1,2,0)}$ & .5 & .4 & 0 & .1 \\
\hline$\beta_{(2,0,1)}$ & .7 & 0 & .2 & .1 \\
\hline$\beta_{(1,0,2)}$ & .5 & 0 & .3 & .2 \\
\hline$\beta_{(0,2,1)}$ & 0 & .4 & .2 & .4 \\
\hline$\beta_{(0,1,2)}$ & 0 & .3 & .3 & .4 \\
\hline
\end{tabular}

Additionally, $\beta_{(3,0,0)}, \beta_{(0,3,0)}$, and $\beta_{(0,0,3)}$ assign probability 1 to $a, b$, and $c$, respectively. The associated $\mathrm{PR}$ is denoted by $\psi$, and we will show that $\psi$ cannot be written as a convex combination of unanimous and strategy-proof deterministic rules.

Let $F$ be the set of all unanimous and strategy-proof deterministic rules for preference profiles that are single-peaked with respect to the given tree. Further, for an alternative $x$ and a profile $P_{N}$, let $F\left(x, P_{N}\right)$ be the set of all deterministic rules $f$ such that 
$f\left(P_{N}\right)=x$. By $\left(S_{1}, S_{2}, S_{3}\right)$, where $S_{1}, S_{2}, S_{3}$ are disjoint with union $N$, we denote a profile where the top-alternatives of the agents in $S_{1}, S_{2}$, and $S_{3}$ are $a, b$, and $c$, respectively. Let $F_{1}=F(a,(\{1,2\},\{3\}, \emptyset)), F_{2}=F(b,(\{1,3\},\{2\}, \emptyset)), F_{3}=F(c,(\{1\},\{2\},\{3\}))$, $F_{4}=F(b,(\{1,2\},\{3\}, \emptyset))$, and $F_{5}=F(b,(\{1\},\{2,3\}, \emptyset))$. Then, by Theorem 3.13, or more directly by uncompromisingness (Lemma 3.2), it follows that $F_{1} \cap F_{3}=\emptyset$ and $F_{2} \cap F_{3}=\emptyset$. Combining, we have

$$
\left(F_{1} \cup F_{2}\right) \cap F_{3}=\emptyset .
$$

Assume for contradiction that $\psi$ can be written as $\sum_{f \in F} \alpha_{f} f$, where $\alpha_{f} \geq 0$ for all $f \in F$ and $\sum_{f \in F} \alpha_{f}=1$. For $G \subseteq F$, let $\alpha_{G}=\sum_{f \in G} \alpha_{f}$. Then $\alpha_{F_{1} \cup F_{2}}=\alpha_{F_{1}}+\alpha_{F_{2}}-\alpha_{F_{1} \cap F_{2}}$ together with (25), yields $\alpha_{F_{1}}+\alpha_{F_{2}}-\alpha_{F_{1} \cap F_{2}}+\alpha_{F_{3}} \leq 1$. Since $\psi=\sum_{f \in F} \alpha_{f} f$, we have $\alpha_{F_{1}}=\psi_{a}(\{1,2\},\{3\}, \emptyset), \alpha_{F_{2}}=\psi_{b}(\{1,3\},\{2\}, \emptyset), \alpha_{F_{3}}=\psi_{c}(\{1\},\{2\},\{3\})$. Using the values given in the table we obtain

$$
\alpha_{F_{1} \cap F_{2}} \geq 0.2 \text {. }
$$

Since the rules in $F_{1}$ and $F_{4}$ give different outcomes ( $a$ and $b$, respectively) at the same profile $(\{1,2\},\{3\}, \emptyset)$, we have $F_{1} \cap F_{4}=\emptyset$. Moreover, by uncompromisingness, $F_{2} \subseteq F_{5}$ and $F_{4} \subseteq F_{5}$, and hence $F_{2} \cup F_{4} \subseteq F_{5}$. Because $F_{1} \cap F_{4}=\emptyset$, we have

$$
\left(F_{1} \cap F_{2}\right) \cap F_{4}=\emptyset \text {. }
$$

Also, because $F_{2} \cup F_{4} \subseteq F_{5}$,

$$
\left(F_{1} \cap F_{2}\right) \cup F_{4} \subseteq F_{5} .
$$

Combining (27) and (28), we have $\alpha_{F_{1} \cap F_{2}}+\alpha_{F_{4}} \leq \alpha_{F_{5}}$. By (26) and the table, $\alpha_{F_{1} \cap F_{2}}+\alpha_{F_{4}} \geq$ 0.5 , and hence $\alpha_{F_{5}} \geq 0.5$. However, from the table it follows that $\alpha_{F_{5}}=0.4$. This is a contradiction. Thus, $\psi$ cannot be written as a convex combination of deterministic rules.

\section{Concluding remarks}

The main result in this paper (Theorem 5.5) characterizes all unanimous and strategyproof probabilistic rules for single-peaked preference profiles on a connected but otherwise arbitrary graph of which the nodes are the alternatives. Such a rule is a random dictatorship on the maximal leafless subgraph, and on each branch it is a monotonic rule - extending the median-like rules in Moulin (1980) and the probabilistic rules in Ehlers et al (2002) on the line graph - such that the total probability on each branch equals the sum of the random dictatorship weights of the agents who have their peaks on this branch.

Contrary to the line graph case (Peters et al, 2014) not every probabilistic rule is a convex combination of deterministic rules, as we have shown in Section 6.

An earlier version of the paper (Peters et al, 2018) shows that at least the results on the case where the graph is a tree can be derived for a smaller set of single-peaked preferences.

\section{A Proof of Lemma 2.6}

The proof of Lemma 2.6 will be based on Theorem 1 in Chatterji and Zeng (2018). We need to introduce two concepts used there, namely the Interior Property and the Exterior Property.

We say that preferences $P, P^{\prime} \in \mathbb{L}(A)$ are adjacent if there are distinct $x, y \in A$ with $P=\cdots x y \cdots$ and $P^{\prime}=\cdots y x \cdots$ such that for all $a, b \in A$ with $\{a, b\} \neq\{x, y\}$ we have 
$a P b \Leftrightarrow a P^{\prime} b$. A subset $\mathcal{L} \subseteq \mathbb{L}(A)$ has the Interior Property if for all $a \in A$ and all $P, P^{\prime} \in \mathcal{L}$ with $t(P)=t\left(P^{\prime}\right)=a$ there are $P^{1}, \ldots, P^{k} \in \mathcal{L}$ with $k \geq 1$ and $t\left(P^{j}\right)=a$ for every $j=1, \ldots, k$ such that $P=P^{1}, P^{\prime}=P^{k}$, and for each $j=1, \ldots, k-1$ the preferences $P^{j}, P^{j+1}$ are adjacent.

Lemma A.1. Let $G=(A, E)$ be a connected graph. Then $\mathcal{S}$ has the Interior Property.

Proof. Let $1 \leq k \leq|A|-2$ and let $a_{1}, \ldots, a_{k}, a_{k+1}$ be distinct alternatives. Consider a preference $P=a_{1} \cdots a_{k} \cdots x a_{k+1} \cdots$ single-peaked with respect to a spanning tree $T$ of $G$, and a preference $P^{\prime}=a_{1} \cdots a_{k} a_{k+1} \cdots$ single-peaked with respect to a spanning tree $T^{\prime}$ of $G$. (Thus, $a_{1}, \ldots, a_{k}$ are ranked above all other alternatives at $P$, and $a_{1}, \ldots, a_{k+1}$ are ranked above all other alternatives at $P^{\prime}$.) It is sufficient to prove that there is a spanning tree $\tilde{T}$ with respect to which preference $\tilde{P}=a_{1} \cdots a_{k} \cdots a_{k+1} x \cdots$ (adjacent to $P$ ) is single-peaked. If $x$ is not on the path $\pi=\left[a_{1}, a_{k+1}\right]$ in $T$, then we can simply take $\tilde{T}=T$. Otherwise, we have $\pi=\left[a_{1}, \ldots, x, a_{k+1}\right]$. Let $\pi^{\prime}=\left[a_{1}, \ldots, a_{\ell}, a_{k+1}\right]$ be the path in $T^{\prime}$ from $a_{1}$ to $a_{k+1}$; observe that the alternatives in $\pi^{\prime}$ are a subset of $\left\{a_{1}, \ldots, a_{k+1}\right\}$. Construct $\tilde{T}$ from $T$ as follows. First, delete the edge $\left\{x, a_{k+1}\right\}$ from $T$. This results in two disconnected subtrees with $a_{1}, \ldots, a_{k}$ and $x$ in one subtree and $a_{k+1}$ in the other: this follows from single-peakedness of $P$ with respect to $T$ (if $a_{i}$ for some $2 \leq i \leq k$ would be in the same subtree as $a_{k+1}$, then $a_{k+1}$ would be on the path in $T$ from $a_{1}$ to $a_{i}$ and thus $a_{k+1} P a_{i}$ by single-peakedness, a contradiction). Therefore, by adding the edge $\left\{a_{\ell}, a_{k+1}\right\}$ we obtain a spanning tree $\tilde{T}$. The proof of the lemma is complete if we show that $\tilde{P}$ is single-peaked with respect to $\tilde{T}$.

Suppose this were not the case. Then there are distinct $z, z^{\prime} \in A$ such that $z$ is on the path $\pi=\left[a, z^{\prime}\right]$ in $\tilde{T}$, but $z^{\prime} \tilde{P} z$. If $\pi$ is also a path in $T$, then we have $z P z^{\prime}$, hence $z=x$ and $z^{\prime}=a_{k+1}$, and in particular $\left\{x, a_{k+1}\right\}$ is an edge in $\tilde{T}$, which is a contradiction. Hence, $\pi$ is not a path in $T$, and we can write $\pi=\left[a_{1}, a_{\ell}\right] \cdot\left\{a_{\ell}, a_{k+1}\right\} \cdot\left[a_{k+1}, z^{\prime}\right]$, where $\left[a_{1}, a_{\ell}\right]$ and $\left[a_{k+1}, z^{\prime}\right]$ are also paths in $T$. If $z \in\left[a_{k+1}, z^{\prime}\right]$ then $z$ is on the path $\left[a_{1}, x\right] \cdot\left\{x, a_{k+1}\right\} \cdot\left[a_{k+1}, z^{\prime}\right]$ in $T$, hence $z P z^{\prime}$ and therefore $z \tilde{P} z^{\prime}$, a contradiction. Therefore, we have that $z$ is on the path $\left[a_{1}, a_{\ell}\right]$ in $T$ and $\tilde{T}$, thus $z \in\left\{a_{1}, \ldots, a_{k}\right\}$, and again $z \tilde{P} z^{\prime}$, a contradiction.

For a preference $P \in \mathbb{L}(A)$ and a number $\ell \in\left\{1, \ldots, \leq|A|\right.$, let $B_{\ell}(P) \subseteq A$ denote the set of the $\ell$ highest ranked alternatives according to $P$, i.e., if $P=a_{1} \cdots a_{\ell} a_{\ell+1} \cdots a_{|A|}$ then $B_{\ell}(P)=\left\{a_{1}, \ldots, a_{\ell}\right\}$. A subset $\mathcal{L} \subseteq \mathbb{L}(A)$ has the Exterior Property if for all $P, P^{\prime} \in \mathcal{L}$ with $t(P) \neq t\left(P^{\prime}\right)$ and all distinct $x, y \in A$ with $x P y$ and $x P^{\prime} y$, there are $P^{1}, \ldots, P^{k} \in \mathcal{L}, k \geq 2$, such that $P=P^{1}, P^{\prime}=P^{k}$, and for every $j=1, \ldots, k-1$ there is an $\ell \in\{1, \ldots, \leq|A|$ such that $x \in B_{\ell}\left(P^{j}\right)=B_{\ell}\left(P^{j+1}\right)$ and $y \notin B_{\ell}\left(P^{j}\right)$.

Lemma A.2. Let $G=(A, E)$ be a connected graph. Then $\mathcal{S}$ has the Exterior Property.

Proof. Let $P, P^{\prime} \in \mathcal{S}$ with $t(P)=a \neq b=t\left(P^{\prime}\right)$ and distinct $x, y \in A$ with $x P y$ and $x P^{\prime} y$. Let $T$ be a spanning tree of $G$ with respect to which $P$ is single-peaked.

(i) First suppose that $b P y$. Let the path $[a, b]$ in $T$ consist of the sequence $a, z_{1}, \ldots, z_{k}, b$, hence $a P z_{1} \ldots P z_{k} P b$. Define $P^{\prime \prime}=b z_{k} \cdots z_{1} a \cdots$ such that $z P^{\prime \prime} z^{\prime} \Leftrightarrow z P z^{\prime}$ for all $z, z^{\prime} \in$ $A \backslash\left\{a, z_{1}, \ldots, z_{k}, b\right\}$, and let $\ell=\max \{|U(b, P)|,|U(x, P)|\}$.

We show that $P^{\prime \prime}$ is single-peaked with respect to $T$. To this end, let $\left[b \cdots z \cdots z^{\prime}\right]$ be a path in $T$. We show that $z P^{\prime \prime} z^{\prime}$. If $z, z^{\prime} \in\left\{a, z_{1}, \ldots, z_{k}, b\right\}$, say $z=z_{i}$ and $z^{\prime}=z_{j}$, then we have $i>j$ and $z_{i} P^{\prime \prime} z_{j}$, hence $z P^{\prime \prime} z^{\prime}$. If $z \in\left\{a, z_{1}, \ldots, z_{k}, b\right\}$ and $z^{\prime} \notin\left\{a, z_{1}, \ldots, z_{k}, b\right\}$ then $z P^{\prime \prime} z^{\prime}$. If $z \notin\left\{a, z_{1}, \ldots, z_{k}, b\right\}$ and $z^{\prime} \in\left\{a, z_{1}, \ldots, z_{k}, b\right\}$ then $\left[b \cdots z \cdots z^{\prime}\right] \cdot\left[z^{\prime} \cdots b\right]$ contains a cycle, a contradiction. If, finally, $z, z^{\prime} \notin\left\{a, z_{1}, \ldots, z_{k}, b\right\}$ then there is a path $\left[a \cdots z \cdots z^{\prime}\right]$ 
in $T$, hence $z P z^{\prime}$ and therefore $z P^{\prime \prime} z^{\prime}$. This completes the proof of single-peakedness of $P^{\prime \prime}$ with respect to $T$.

Also, $t\left(P^{\prime \prime}\right)=b, x \in B_{\ell}(P)=B_{\ell}\left(P^{\prime \prime}\right)$, and $y \notin B_{\ell}(P)$. The proof for this case is then complete by constructing a sequence of adjacent preferences starting from $P^{\prime \prime}$ and ending in $P^{\prime}$ by using the Interior Property (Lemma A.1).

(ii) Second suppose that $y P b$ and $y$ is not on the path $[a, b]$ in $T$. Construct the preference $\tilde{P}$ as follows. Let $C=\{z \in A: y$ is on the path $[a, z]$ in $T\}$. Then let $z^{\prime} \tilde{P} z$ for all $z \in C$ and $z^{\prime} \in A \backslash C$, and $z \tilde{P} z^{\prime} \Leftrightarrow z P z^{\prime}$ for all $z, z^{\prime} \in C$ and all $z, z^{\prime} \in A \backslash C$. Then $\tilde{P}$ is still single-peaked with respect to $T$, and for $\ell=|U(x, P)|$ we have $x \in B_{\ell}(P)=B_{\ell}(\tilde{P})$ and $y \notin B_{\ell}(P)$. Since $b \notin C$ and therefore $b \tilde{P} y$ we can complete the proof by applying the arguments in (i) now starting from $\tilde{P}$.

(iii) Third suppose that $y P b$ and $y$ is on the path $[a, b]$ in $T$. Write $a \cdots a^{\prime} y \cdots b$ for the path $[a, b]$ in $T$. Let, similarly as above, $C=\{z \in A: y$ is on the path $[a, z]$ in $T\}$. Since $P^{\prime} \in \mathcal{S}$ there is a path $\pi=[b, x]$ in $G$ with $y \notin[b, x]$. On this path let $\{c, d\}$ be the first edge with $c \in C$ and $d \in A \backslash C$. Now first delete the edge $\left\{a^{\prime}, y\right\}$ from $T$; next add the part $\pi^{\prime}=[b \cdots c d]$ of $\pi$; and finally delete edges $\{v, w\}$ with $v, w \in C$ but $\{v, w\}$ not in $\pi^{\prime}$ such that a spanning tree $\bar{T}$ of $G$ is obtained. Next construct a preference $\bar{P}$, single-peaked with respect to $\bar{T}$, with $z \bar{P} z^{\prime}$ for all $z \in A \backslash C$ and $z \in C, z \bar{P} z^{\prime} \Leftrightarrow z P z^{\prime}$ for all $z, z^{\prime} \in A \backslash C$, $x \in B_{p}(P)=B_{p}\left(\bar{P}\right.$, and $y \notin B_{p}(P)$, where $p=|U(x, P)|=|U(x, \bar{P})|$. Then either $b \bar{P} y$, and we are back in case (i), or $y \bar{P} b$. In the latter case, since the path $[a, b]$ in $\bar{T}$ is of the form $[a \cdots d c \cdots b]$ where $[d c \cdots b]$ is the converse path of $\pi^{\prime}, y(\in C)$ is not on this path, and we are back in case (ii).

Lemma 2.6 now follows by applying Theorem 1 in Chatterji and Zeng (2018).

\section{References}

Barberà S, Gul F, Stacchetti E (1993) Generalized median voter schemes and committees. Journal of Economic Theory 61:262-289

Black D (1948) On the rationale of group decision-making. Journal of Political Economy $56: 23-34$

Bondy JA, Murty USR (2008) Graph Theory. Springer, Heidelberg

Border K, Jordan J (1983) Straightforward elections, unanimity and phantom voters. Review of Economic Studies 50:153-170

Chatterji S, Massó J (2018) On strategy-proofness and the salience of single-peakedness. International Economic Review 59:163-189

Chatterji S, Sen A, Zeng H (2014) Random dictatorship domains. Games and Economic Behavior 86:212-236

Chatterji S, Sen A, Zeng H (2016) A characterization of single-peaked preferences via random social choice functions. Theoretical Economics 11:711-733

Chatterji S, Zeng H (2018): On random social choice functions with the tops-only property. Games and Economic Behavior 109:413-435 
Demange G (1982) Single peaked orders on a tree. Mathematical Social Sciences 3:389-396

Dutta B, Peters H, and Sen A (2002) Strategy-Proof probabilistic mechanisms in economies with pure public goods. Journal of Economic Theory 106:392-416

Dutta B, Peters H, Sen A (2007) Strategy-proof cardinal decision schemes. Social Choice and Welfare 28:163-179. Erratum (2008). Social Choice and Welfare 30:701-702

Ehlers L, Peters H, Storcken A (2002) Strategy-proof probabilistic decision schemes for one-dimensional single-peaked preferences. Journal of Economic Theory 105:408-434

Gibbard A (1977) Manipulation of voting schemes that mix voting with chance. Econometrica 45:665-681

Hansen P, Thisse JF (1981) Outcomes of voting and planning: Condorcet, Weber, and Rawls locations. Journal of Public Economics 16:1-5

Hylland A (1980) Strategy proofness of voting procedures with lotteries as outcomes and infinite sets of strategies. University of Oslo, Institute of Economics

Inada KI (1964) A note on the simple majority rule. Econometrica 32:525-531

Menger K (1927) Zur allgemeinen Kurventheorie. Fund. Math. 10:46-68

Moulin H (1980) On strategy-proofness and single peakedness. Public Choice 35:437-455

Nandeibam S (2013) The structure of decision schemes with cardinal preferences. Review of Economic Design 17:205-238

Peters H, Roy S, Sadhukhan S (2018) Random social choice functions for single-peaked domains on trees. GSBE Research Memorandum 18/004, Maastricht

Peters H, Roy S, Sen A, Storcken T (2014) Probabilistic strategy-proof rules over singlepeaked domains. Journal of Mathematical Economics 52:123-127

Schummer J, Vohra RV (2002) Strategy-proof location on a network. Journal of Economic Theory 104:405-428

Sen A (2011) The Gibbard random dictatorship theorem: A generalization and a new proof. Spanish Economic Review, SERIEs 2(4):515-527

Whitney H (1931) Non-separable and planar graphs. Proceedings of the National Academy of Sciences of the United States of America 17:125-127 